\title{
Large impact parameter behavior in the CGC/saturation approach: A new nonlinear equation
}

\author{
E. Gotsman ${ }^{1, *}$ and E. Levin ${ }^{1,2, \dagger}$ \\ ${ }^{1}$ Department of Particle Physics, School of Physics and Astronomy, \\ Raymond and Beverly Sackler Faculty of Exact Science, \\ Tel Aviv University, Tel Aviv 69978, Israel \\ ${ }^{2}$ Departamento de Física, Universidad Técnica Federico Santa María and Centro Científico-Tecnológico \\ de Valparaíso, Casilla 110-V, Valparaiso, Chile
}

(Received 6 November 2019; published 28 January 2020)

\begin{abstract}
In this paper we propose a solution to the long standing problem present in the color glass condensate/ saturation approach: i.e., the powerlike fall off of the scattering amplitudes at large $b$. We propose a new nonlinear equation, which takes into account random walks both in transverse momenta of the produced gluons, and in their impact parameter. We demonstrate, that this equation is in accord with previous attempts to include diffusion in impact parameters in the Balitsky-Fadin-Kuraev-Lipatov evolution equation. We show in the paper, that the solution to a new equation results in the exponential decrease of the scattering amplitude at large impact parameter, and in the restoration of the Froissart theorem.
\end{abstract}

DOI: 10.1103/PhysRevD.101.014023

\section{INTRODUCTION}

It is well known that perturbative QCD has a fundamental problem: the scattering amplitude decreases at large impact parameters $(b)$ as a power of $b$. In particular, the color glass condensate (CGC)/saturation approach [1], which is based on perturbative QCD, also has this problem. At large $b$ the scattering amplitude is small and, therefore in perturbative QCD, only the linear Balitsky-Fadin-KuraevLipatov (BFKL) equation [2] is determined by the scattering amplitude. It is known that the eigenfunction of this equation (the scattering amplitude of two dipoles with sizes $r$ and $R$ ) has the following form [3]

$$
\begin{aligned}
\phi_{\gamma}(\boldsymbol{r}, \boldsymbol{R}, \boldsymbol{b}) & =\left(\frac{r^{2} R^{2}}{\left(\boldsymbol{b}+\frac{1}{2}(\boldsymbol{r}-\boldsymbol{R})\right)^{2}\left(\boldsymbol{b}-\frac{1}{2}(\boldsymbol{r}-\boldsymbol{R})\right)^{2}}\right)^{\gamma} \\
& \stackrel{b \gg r, R}{\longrightarrow}\left(\frac{r^{2} R^{2}}{b^{4}}\right)^{\gamma} \equiv e^{\gamma \xi} \text { with } \xi=\ln \left(\frac{r^{2} R^{2}}{b^{4}}\right)
\end{aligned}
$$

One can see that at large impact parameter $b$, the amplitude has a powerlike decrease, which leads to the violation of the Froissart theorem [4]. The violation of the Froissart theorem stems from the growth of the radius of interaction as a power of the energy. Since in Ref. [3] it was proven that

\footnotetext{
*gotsman@post.tau.ac.il

leving@ tauex.tau.ac.il,eugeny.levin@usm.cl
}

Published by the American Physical Society under the terms of the Creative Commons Attribution 4.0 International license. Further distribution of this work must maintain attribution to the author(s) and the published article's title, journal citation, and DOI. Funded by SCOAP ${ }^{3}$. the eigenfunction of any kernel with conformal symmetry has the form of Eq. (1), one can only change the large $b$ behavior by introducing a new dimensional scale in the kernel of the equation. This problem has been known from the beginning of the saturation approach [5,6], and several ideas have been proposed, of how to introduce a new dimensional scale in the kernel of the BFKL equation (See Refs. [5-10]). However, for the high energy community at large, the problem was appreciated only after the papers of Refs. $[11,12]$ were published, where it was demonstrated, that the violation of the Froissart theorem cannot be avoided in the framework of the CGC approach.

First, we wish to illustrate why the Froissart theorem is violated for the BFKL equation. The general solution to the BFKL for the dipole scattering amplitude equation, has the following form:

$$
\begin{aligned}
N(r, Y ; b) & =\int_{\epsilon-i \infty}^{\epsilon+i \infty} \frac{d \gamma}{2 \pi i} e^{\omega\left(\bar{\alpha}_{S}, \gamma\right) Y} \phi_{\gamma}(\boldsymbol{r}, \boldsymbol{R}, \boldsymbol{b}) \phi_{\mathrm{in}}(\gamma) \\
& =\int_{\epsilon-i \infty}^{\epsilon+i \infty} \frac{d \gamma}{2 \pi i} e^{\omega\left(\bar{\alpha}_{S}, \gamma\right) Y+\gamma \xi} \phi_{\mathrm{in}}(\gamma)
\end{aligned}
$$

where

$$
\begin{aligned}
\omega\left(\bar{\alpha}_{S}, \gamma\right) & =\bar{\alpha}_{S} \chi(\gamma)=\bar{\alpha}_{S}(2 \psi(1)-\psi(\gamma)-\psi(1-\gamma)) \\
& \stackrel{\gamma \rightarrow \frac{1}{2}}{\longrightarrow} \omega_{0}+D\left(\gamma-\frac{1}{2}\right)^{2}+\mathcal{O}\left(\left(\gamma-\frac{1}{2}\right)^{3}\right) \\
& =\bar{\alpha}_{S} 4 \ln 2+\bar{\alpha}_{S} 14 \zeta(3)\left(\gamma-\frac{1}{2}\right)^{2}+\mathcal{O}\left(\left(\gamma-\frac{1}{2}\right)^{3}\right)
\end{aligned}
$$

$\psi(z)$ denotes the Euler psi-function $\psi(z)=d \ln \Gamma(z) / d z$. 
The main contribution in Eq. (2) stems from $\gamma \rightarrow \frac{1}{2}$, where we can use the expansion shown in Eq. (3), evaluating this integral using the method of steepest descend, one can see that saddle point occurs at $\gamma_{\mathrm{SP}}=$ $\frac{1}{2}-\frac{\xi}{2 D Y} \ll 1$ and the amplitude is equal to

$N(r, Y ; b) \stackrel{b \gg r, R}{\longrightarrow} \phi\left(\frac{1}{2}\right)\left(\frac{r^{2} R^{2}}{b^{4}}\right)^{1 / 2} \frac{1}{2 \sqrt{D Y}} e^{\omega_{0} Y-\frac{\xi^{2}}{4 D Y}}$.

Using Eq. (4) we attempt to determine the upper bound from the unitarity constraints

$$
2 N(r, Y ; b)=|N(r, Y ; b)|^{2}+G_{\text {in }}\left(r^{2}, Y ; b\right)
$$

where $G_{\text {in }}$ describes the contribution of all inelastic processes. Recalling that $N$ is the imaginary part of the scattering amplitude and assuming that the real part of the amplitude is small, as is the case of the BFKL equation, the unitarity constraint has the solution:

$$
\begin{aligned}
N & =1-\exp (-\Omega(r, Y ; b))<1 ; \\
G_{\text {in }} & =1-\exp (-2 \Omega(r, Y ; b))
\end{aligned}
$$

where $\Omega>0$, denotes an arbitrary function.

We can find the bound for the total cross section following Ref. [4].

$$
\sigma_{\mathrm{tot}}=2 \int N(r, Y ; b) d^{2} b<2 \int^{b_{0}} d^{2} b+\int_{b_{0}} d^{2} b N(r, Y ; b) .
$$

We need to solve the following equation to find the value of $b_{0}$,

$$
\begin{aligned}
N\left(r, Y ; b_{0}\right) & =\phi\left(\frac{1}{2}\right)\left(\frac{r^{2} R^{2}}{b^{4}}\right)^{1 / 2} \frac{1}{2 \sqrt{D Y}} e^{\omega_{0} Y-\frac{\xi^{2}}{4 D Y}} \\
& =f_{0}<1 .
\end{aligned}
$$

At large $Y$ this solution gives $b_{0}^{2} \propto e^{\left(D+\sqrt{D\left(D+4 \omega_{0}\right)}\right) Y}$, and therefore,

$$
N\left(r, Y ; b_{0}\right)<e^{\left(D+\sqrt{D\left(D+4 \omega_{0}\right)}\right)} .
$$

Note, that for the soft amplitude of the typical form $N_{\text {soft }} \propto e^{\omega_{0} Y-\mu b}$ and $b_{0} \propto Y$. In this case, one can see, that the first integral in Eq. (7) leads to $\sigma \leq$ Const $y^{2}$, which is the Froissart theorem. This amplitude violates the Froissart theorem and can be considered only at large values of $b$ where $N_{\text {soft }}<1$. However, the eikonal solution of Eq. (6) with $\Omega=N_{\text {soft }}$, satisfies the unitarity constraints and leads to the amplitude, that describes the Froissart disc: the amplitude is equal to 1 for $b<(1 / \mu) Y$ and it has an edge which behaves as $N_{\text {soft }}$.
We hope that this simple estimate shows, that the powerlike decrease of the scattering amplitude is the source of the problem. To solve the problem in the framework of the CGC/saturation approach we need to introduce in addition to the saturation momentum, a new dimensional scale $\mu$. In Ref. [4] it is shown that this new scale is related to the mass of the lightest hadrons. Perturbative QCD cannot reproduce the observed spectrum of hadrons, and attempting to solve this problem, we are doomed to introduce something from nonperturbative QCD estimates. Since the nonperturbative approach is still in an embryonic stage, one can only make a guess of how to introduce this scale, which depends crucially on nonperturbative estimates in lattice $\mathrm{QCD}$, on the phenomenology of high energy interactions and on intuition, which comes from considering different theoretical models. We hope that the following is a more or less complete list of the attempts to solve this problem, and to find and introduce a new dimensional scale appear in Refs. [5-10,12-24].

At first sight the recent papers $[25,26]$ have questioned the need of a new dimensional scale, since they demonstrate that the next-to-leading order BFKL equation generates the exponential type of the impact parameter behavior without the need of a new dimensional scale. However, it turns out [27] that the NLO corrections do not change the powerlike decrease of the scattering amplitude at large impact parameter, generating the exponential-type decreases in the large but limited region of the values of the impact parameter. ${ }^{1}$

In this paper we re-visit one of the possible ways of introducing a new dimensional scale: to incorporate in the BFKL equation the diffusion in impact parameter $(b)$. The first such attempt was undertaken in the distant 1990's [5,6] and during three decades we have worked in this area [7-10,14,23,24]. In the preprint version of this paper [28] we give an extended review of all efforts to introduce a new dimensional scale in the CGC framework. We consider this review as the important part of presentation, which helps a reader to trust our generalization of the BFKL equation, since it shows that the Green function of the BFKL Pomeron, that follows from our new equation, coincides with other more phenomenological ways of introducing a new dimensional scale. We were advised, that the manuscript would be improved, if our historical review is shortened. Therefore, after discussion of two kind of diffusions: in impact parameter and in transverse momenta in QCD in the next section, we propose in Sec. III our generalization of the BFKL equation, which takes into account the diffusion in $b$ in accord with QCD estimates. We will discuss the structure of the scattering amplitude at

\footnotetext{
${ }^{1}$ In addition to the powerlike behavior at large $b$ the NLO corrections lead to an oscillating behavior of the scattering amplitude at large $b$, in direct contradiction with the unitarity constraint [27].
} 
high energies, which at the present appears to be a black disc with radius increasing as a power of energy. This paper is partly motivated by Ref. [24], in which many questions concerning QCD has been formulated on the black disc behavior at high energy ${ }^{2}$ from the point of boost invariance and the parton model. We hope that we succeed in answering some of these questions in this paper. In particular, we will demonstrate in Sec. IV, that QCD leads to the Froissart disc at high energies, with specific behavior of the amplitude at the edge of this disc.

\section{DIFFUSIONS}

\section{A. Regge approach and Gribov's diffusion in impact parameter}

In the framework of the Regge approach the high energy amplitude is given by the exchange of the Pomeron, and has the following form [29-32]:

$s N\left(s, Q_{t}\right) \equiv \operatorname{Im} A\left(s, Q_{T}\right)=g_{1}\left(Q_{T}\right) g_{2}\left(Q_{T}\right) e^{\alpha_{\mathbb{P}}\left(Q_{T}^{2}\right) Y}$

where $g_{1}, g_{2}$ and trajectory $\alpha_{\mathbb{P}}\left(Q_{T}^{2}\right) \equiv 1+\Delta_{\mathbb{P}}\left(Q_{T}\right)=$ $1+\Delta_{\mathbb{P}}-\alpha_{\mathbb{P}}^{\prime} Q_{T}^{2}+\mathcal{O}\left(Q_{T}^{4}\right)$ are functions that have to be taken from phenomenology. $Y=\ln (s)$ and $Q_{T}$ is the momentum transferred by the Pomeron. ${ }^{3}$ Equation (10) can be viewed as the solution to the following equation:

$$
\frac{d N\left(Y, Q_{T}\right)}{d Y}=\Delta_{\mathbb{P}}\left(Q_{T}\right) N\left(Y, Q_{T}\right)
$$

with the initial condition

$$
N\left(Y=0, Q_{T}\right)=g_{1}\left(Q_{T}\right) g_{2}\left(Q_{T}\right) .
$$

In impact parameter representation the solution of Eq. (10) takes the form:

$$
\begin{gathered}
N(Y, b)=\int \frac{d^{2} Q_{T}}{(2 \pi)^{2}} e^{i b \cdot Q_{T}} N\left(Y=0, Q_{T}\right)=g_{1} g_{2} e^{\Delta_{\mathbb{P}}, Y} n(Y, b) \\
\text { with } n(Y, b)=\frac{1}{4 \pi \alpha_{\mathbb{P}}^{\prime} Y} e^{-\frac{b^{2}}{4 \alpha_{\mathbb{P}}^{\prime} Y}} .
\end{gathered}
$$

In Eq. (13) we have neglected the $Q_{T}$ dependence of $g_{1}$ and $g_{2}$ which do not contribute at high energies.

In Ref. [31] the simple fact is noted, i.e., $n(Y, b)$ is the solution of the diffusion equation:

$$
\frac{d n(Y, b)}{d Y}=\alpha_{\mathbb{P}}^{\prime} \nabla_{b}^{2} n(Y, b)
$$

\footnotetext{
${ }^{2}$ We will use the Froissart disc instead the black disc behavior with the radius which increase as $\ln (s)$.

${ }^{3}$ In the case of the deep inelastic processes $Y=\ln (1 / x)$, where $x$ is the Bjorken variable.
}

Equation (14) together with Eq. (10) for the total cross section:

$\sigma_{\mathrm{tot}}=2 g_{1} g_{2} N\left(Y, Q_{T}=0\right)=2 g_{1} g_{2} e^{\Delta_{\mathrm{P}} Y}=\sigma_{0} \sum_{n=0}^{\infty} \frac{\Delta_{\mathbb{P}} Y}{n !}$

have very simple interpretations in the parton model. In the parton model [31-33] it is assumed, that we can describe the interaction by a field theory, in which all integrals over transverse momenta are convergent, and they lead to the mean transverse momentum, which does not depend on energy. In such a theory, the contribution to the total cross section of the scattering amplitude for production of $n$ partons in each order of perturbation approach, can be viewed as

$$
\begin{aligned}
\sigma_{n} & =M_{2 \rightarrow 2+n}\left(\left\{p_{i, T}\right\}\right) \prod_{i=0}^{n} d^{2} p_{i, T} d y_{i} \\
& =M_{2 \rightarrow 2+n}\left(\left\{p_{i, T}\right\}\right) \prod_{i=0}^{n} d^{2} p_{i, T} \frac{Y^{n}}{n !} .
\end{aligned}
$$

In Eq. (16) we assume that in the proposed theory the amplitude is not equal to zero, when rapidities of emitted partons are equal to zero, and choose the largest contribution which comes from the ordering

$$
0<y_{1}<y_{2}<\ldots<y_{i}<\ldots<y_{n}<Y \text {. }
$$

One can see that in Eq. (15) $M_{2 \rightarrow 2+n}\left(\left\{p_{i, T}\right\}\right) \prod_{i=0}^{n} d^{2} p_{i, T}=$ $\Delta_{\mathbb{P}}^{n^{4}}$ and from this equation we can conclude that the number of emitted partons $n=\Delta_{\mathbb{P}} Y$. Gribov's idea, that the emission of partons has no other correlations except the fixed transverse momentum, can be viewed as a random walk in two dimensional space. For each emission due to uncertainty principle

$$
\Delta b_{i} p_{i, T} \sim 1 \quad \text { or } \quad \Delta b_{i} \sim \frac{1}{\left\langle\left|p_{i, T}\right|\right\rangle} .
$$

Therefore, after each emission the position of the parton will be shifted by an amount $\Delta b^{2}$ from Eq. (18), which on average is the same. After $n$ emissions, we have the picture shown in Fig. 1, and the total shift in $b$ is equal to

$$
R_{\mathrm{int}}^{2}=b_{n}^{2}=\frac{1}{\left\langle\left|p_{i, T}^{2}\right|\right\rangle}=\frac{1}{\left\langle\left|p_{i, T}^{2}\right|\right\rangle} \Delta_{\mathbb{P}} Y
$$

Therefore, this diffusion reproduces the shrinkage of the diffraction peak. Indeed,

\footnotetext{
${ }^{4}$ In this estimate we assume that $\sigma_{0}$ in Eq. (13) does not depend on energy. In the field theories which can be a realization of the parton model, usually $\sigma_{0} \propto 1 / s^{2}$ but the first result from QCD was the understanding that in this approach $\sigma_{0}$ does not depend on energy [34].
} 


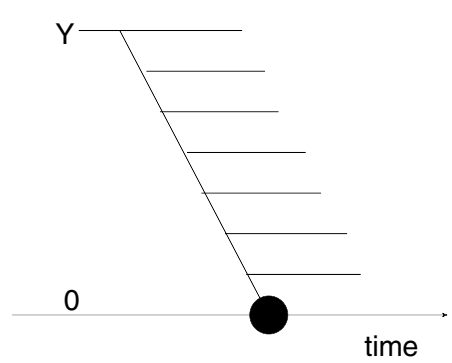

(a)

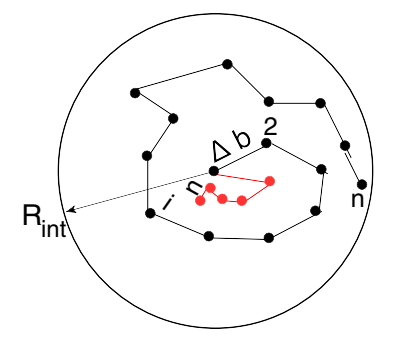

(b)
FIG. 1. The structure of the parton cascade: Fig. 1(a) shows the time structure of the cascade while Fig. 1(b) illustrates the random walk in $b$. The black lines describe the diffusion in the parton model, while the red lines correspond to a random walk in $b$ for QCD.

$$
R^{2}=\frac{\int d^{2} b b^{2} N(Y, b)}{\int d^{2} b N(Y, b)}=4 \alpha_{\mathbb{P}}^{\prime} Y
$$

Comparing Eq. (19) and Eq. (20) one can see that

$$
\alpha_{\mathbb{P}}^{\prime}=\frac{\Delta_{\mathbb{P}}}{4\left\langle\left|p_{i, T}^{2}\right|\right\rangle} .
$$

Equation (11) can be rewritten in the impact parameter representation for $N(Y, b)$ of Eq. (13)

$$
\begin{aligned}
\frac{d N(Y, b)}{d Y}= & \int d^{2} b^{\prime} K\left(\boldsymbol{b}-\boldsymbol{b}^{\prime}\right) N\left(Y, b^{\prime}\right) \\
& \text { where } K(b)=\int \frac{d^{2} Q_{T}}{(2 \pi)^{2}} e^{i \boldsymbol{b} \cdot \boldsymbol{Q}_{T}} \Delta_{\mathbb{P}}\left(Q_{T}\right)
\end{aligned}
$$

Writing the equation for the radius of interaction [see Eq. (20)]. First, we see that for $\int d^{2} b b^{2} N(Y, b)=$ $R^{2} \int d^{2} b N(Y, b)$ we have the following equation:

$$
\begin{aligned}
\frac{d\left(R^{2} \int d^{2} b N(Y, b)\right)}{d Y} & =\int d^{2} b d^{2} b^{\prime} b^{2} K\left(\boldsymbol{b}-\boldsymbol{b}^{\prime}\right) N\left(Y, b^{\prime}\right) \\
& =\int d^{2}\left(\boldsymbol{b}-\boldsymbol{b}^{\prime}\right) d^{2} b^{\prime} K\left(\boldsymbol{b}-\boldsymbol{b}^{\prime}\right)\left(\left(\boldsymbol{b}-\boldsymbol{b}^{\prime}\right)^{2}+2\left(\boldsymbol{b}-\boldsymbol{b}^{\prime}\right) \cdot \boldsymbol{b}^{\prime}+b^{\prime 2}\right) N\left(Y, b^{\prime}\right) \\
& =\underbrace{\left(\int d^{2}\left(\boldsymbol{b}-\boldsymbol{b}^{\prime}\right) K\left(\boldsymbol{b}-\boldsymbol{b}^{\prime}\right)\left(\left(\boldsymbol{b}-\boldsymbol{b}^{\prime}\right)^{2}\right)\right.}_{\langle|\Delta b|\rangle \Delta_{\mathbb{P}}\left(Q_{T}=0\right)} \int d^{2} b^{\prime} N\left(Y, b^{\prime}\right)+\underbrace{\int d^{2}\left(\boldsymbol{b}-\boldsymbol{b}^{\prime}\right) d^{2} b^{\prime} K\left(\boldsymbol{b}-\boldsymbol{b}^{\prime}\right)\left(\boldsymbol{b}-\boldsymbol{b}^{\prime}\right) \cdot \boldsymbol{b}^{\prime} N\left(Y, b^{\prime}\right)}_{=0} \\
& +\underbrace{\left(\int d^{2}\left(\boldsymbol{b}-\boldsymbol{b}^{\prime}\right) K\left(\boldsymbol{b}-\boldsymbol{b}^{\prime}\right)\right)}_{\Delta\left(Q_{T}=0\right)}\left(\int d^{2} b^{\prime} b^{\prime 2} N\left(Y, b^{\prime}\right)\right)
\end{aligned}
$$

The second term vanishes due to integration over the angle and finally we have the following equation

$$
\frac{d R^{2}}{d Y}=\Delta_{\mathbb{P}}\left\langle\left|\Delta b^{2}\right|\right\rangle
$$

with the solution $R^{2}=\Delta_{\mathbb{P}}\left\langle\left|\Delta b^{2}\right|\right\rangle Y=-\left.\nabla_{Q_{T}}^{2} \Delta_{\mathbb{P}}\left(Q_{T}\right)\right|_{Q_{T}=0} Y$.

We can obtain Eq. (24) using the Mueller diagrams [35] of Fig. 2.

Indeed, one can see that

$$
\begin{aligned}
& \left.\nabla_{Q_{T}}^{2} N\left(Y, Q_{T}\right)\right|_{Q_{T}=0} \\
& \quad=-\left.\int d Y^{\prime} e^{\Delta_{\mathbb{P}}\left(Y-Y^{\prime}\right)} e^{\left.\Delta_{\mathbb{P}} Y^{\prime}\right)} \nabla_{Q_{T}}^{2} \Delta_{\mathbb{P}}\left(Q_{T}\right)\right|_{Q_{T}=0} \\
& \quad=4 \alpha_{\mathbb{P}}^{\prime} Y e^{\Delta_{\mathbb{P}} Y}
\end{aligned}
$$

Therefore, for $R^{2}$ we obtain $R^{2}=4 \alpha_{\mathbb{P}}^{\prime} Y e^{\Delta_{\mathbb{P}} Y} / e^{\Delta_{\mathbb{P}} Y}=4 \alpha_{\mathbb{P}}^{\prime} Y$ in accord with Eq. (24).
The amplitude of Eq. (13) increases with energy and violates the unitary constraints [see Eq. (5)]. The eikonal unitarization of Eq. (6) leads to the following amplitude

$$
N(Y, b)=1-\exp \left(-e^{\Delta_{\mathbb{P}} Y} \frac{1}{4 \pi \alpha_{\mathbb{P}}^{\prime} Y} e^{-\frac{b^{2}}{4 \alpha_{\mathbb{P}}^{\prime} Y}}\right)
$$

One can see that this amplitude tends to unity at $b>$ $2 \sqrt{\Delta_{\mathbb{P}} \alpha_{\mathbb{P}}^{\prime}} Y$ leading to the total cross section $\sigma_{\text {tot }} \propto Y^{2}$ in accord with the Froissart theorem [4].

In spite of the primitive level of calculations, especially if one compares them with typical QCD calculations in DIS, the parton model has been a good guide for the Pomeron structure for years and, it is still the model where one can see all typical features of the soft Pomeron. It turns out that the time structure of the parton cascade is preserved for QCD and simple parton estimates can help develop our intuition regarding the solution of the QCD problems. 


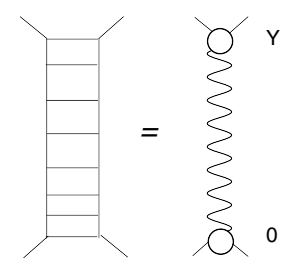

(a)

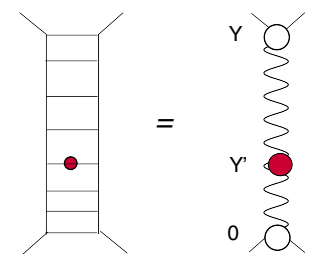

(b)

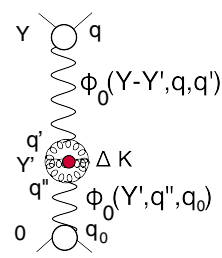

(c)

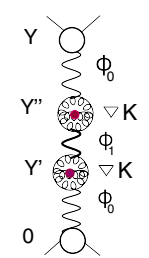

(d)

FIG. 2. Mueller diagrams for calculating $\left.\nabla_{Q_{T}}^{2} N\left(Y, Q_{T}\right)\right|_{Q_{T}=0}$. The wavy lines denote the Pomeron contribution at $Q_{T}=0$. For Fig. 2-a and Fig. 2-b it is the Pomeron in the parton model of Eq. (10), while for Fig. 2(c) and Fig. 2(d) it is the BFKL Pomeron in QCD [see Eq. (33)]. The red blobs denote $\left.\nabla_{Q_{T}}^{2} \Delta_{\mathbb{P}}\left(Q_{T}\right)\right|_{Q_{T}=0}$ or $\left.\nabla_{Q_{T}} \Delta_{\mathbb{P}}\left(Q_{T}\right)\right|_{Q_{T}=0}$ as it is indicated in the figures. Functions $\phi_{0}$ and $\phi_{1}$ are defined in the text.

\section{B. BFKL approach and diffusion in $\ln \left(p_{T}\right)$}

\section{The BFKL equation}

The BFKL equation was derived in momentum representation [2] and has the following form:

$$
\frac{\partial \tilde{N}\left(Y ; q, Q_{T}\right)}{\partial Y}=\bar{\alpha}_{S}\left(\int \frac{d^{2} q^{\prime}}{2 \pi} K_{\mathrm{em}}\left(\boldsymbol{q}-\boldsymbol{q}^{\prime}, \boldsymbol{Q}_{T}\right) \tilde{N}\left(Y ; q^{\prime}, Q_{T}\right)-K_{\mathrm{reg}}\left(\boldsymbol{q}-\boldsymbol{q}^{\prime}, \boldsymbol{Q}_{T}\right) \tilde{N}\left(Y ; q^{\prime}, Q_{T}\right)\right)
$$

where $\bar{\alpha}_{S}=\left(N_{c} / \pi\right) \alpha_{S}$. Kernel $K_{\text {em }}$ describes the emission of a gluon, while kernel $K_{\text {reg }}$ is responsible for the Reggeization of gluons in t-channel. They have the forms:

$$
\begin{aligned}
& K_{\mathrm{em}}\left(\boldsymbol{q}-\boldsymbol{q}^{\prime}, \boldsymbol{Q}_{T}\right)=\frac{1}{2} \frac{1}{\left(\boldsymbol{q}-\boldsymbol{q}^{\prime}\right)^{2}}\left\{-\frac{Q_{T}^{2}\left(\boldsymbol{q}-\boldsymbol{q}^{\prime}\right)^{2}}{\left(\boldsymbol{Q}_{T}-\boldsymbol{q}^{\prime}\right)^{2} q^{\prime 2}}+1+\frac{\left(\boldsymbol{Q}_{T}-\boldsymbol{q}\right)^{2} q^{\prime 2}}{\left(\boldsymbol{Q}_{T}-\boldsymbol{q}^{\prime}\right)^{2} q^{2}}\right\} \stackrel{Q_{T}=0}{\longrightarrow} \frac{1}{\left(\boldsymbol{q}-\boldsymbol{q}^{\prime}\right)^{2}} \\
& K_{\mathrm{reg}}\left(\boldsymbol{q}-\boldsymbol{q}^{\prime}, \boldsymbol{Q}_{T}\right)=\frac{1}{2} \frac{1}{\left(\boldsymbol{q}-\boldsymbol{q}^{\prime}\right)^{2}}\left\{\frac{q^{2}}{\left(\boldsymbol{q}-\boldsymbol{q}^{\prime}\right)^{2}+q^{\prime 2}}+\frac{\left(\boldsymbol{Q}_{T}-\boldsymbol{q}\right)^{2}}{\left(\boldsymbol{q}-\boldsymbol{q}^{\prime}\right)^{2}+\left(\boldsymbol{Q}_{T}-\boldsymbol{q}^{\prime}\right)^{2}}\right\} \stackrel{Q_{T}=0}{\longrightarrow} \frac{1}{\left(\boldsymbol{q}-\boldsymbol{q}^{\prime}\right)^{2}} \frac{q^{2}}{\left(\boldsymbol{q}-\boldsymbol{q}^{\prime}\right)^{2}+q^{\prime 2}}
\end{aligned}
$$

This equation is rewritten in the coordinate representation for the scattering amplitude of a dipole of size $r$, at impact parameter $\boldsymbol{b}[3,36]$ :

$$
\frac{\partial}{\partial Y} N(\boldsymbol{r}, \boldsymbol{b}, Y)=\bar{\alpha}_{S} \int \frac{d^{2} \boldsymbol{r}^{\prime}}{2 \pi} K\left(\boldsymbol{r}^{\prime}, \boldsymbol{r}-\boldsymbol{r}^{\prime} ; \boldsymbol{r}\right)\left(N\left(\boldsymbol{r}^{\prime}, \boldsymbol{b}-\frac{1}{2}\left(\boldsymbol{r}-\boldsymbol{r}^{\prime}\right), Y\right)+N\left(\boldsymbol{r}-\boldsymbol{r}^{\prime}, \boldsymbol{b}-\frac{1}{2} \boldsymbol{r}^{\prime}, Y\right)-N(\boldsymbol{r}, \boldsymbol{b}, Y)\right)
$$

with

$$
K\left(\boldsymbol{r}^{\prime}, \boldsymbol{r}-\boldsymbol{r}^{\prime} ; \boldsymbol{r}\right)=\frac{r^{2}}{r^{\prime 2}\left(\boldsymbol{r}-\boldsymbol{r}^{\prime}\right)^{2}}
$$

In Eq. (29)

$$
\begin{aligned}
N(\boldsymbol{r}, \boldsymbol{b}, Y) & =r^{2} \int \frac{d^{2} q}{2 \pi} e^{i \boldsymbol{q} \cdot \boldsymbol{r}} \tilde{N}(q, b, Y) ; \\
\tilde{N}\left(Y ; q, Q_{T}\right) & =\int \frac{d^{2} b}{(2 \pi)^{2}} e^{i \boldsymbol{Q}_{T} \cdot \boldsymbol{b}} \tilde{N}(q, b, Y) .
\end{aligned}
$$

\section{Solutions and random walk in $\ln \left(p_{T}\right)$}

We have discussed solutions in the coordinate representation [see Eq. (1), Eq. (2), and Eq. (4)]. These solutions are very useful for discussions of the nonlinear corrections, since the unitarity constraints are diagonal for the dipole scattering amplitude [see Eq. (5)]. However, discussing the random walk in $\ln \left(p_{T}\right)$ we need a solution in the momentum representation in which $p_{i, T}$ denote the momenta of produced gluons. Note, that in Eq. (28) $\boldsymbol{q}-\boldsymbol{q}^{\prime}=\boldsymbol{p}_{T}$. For $Q_{T}$ the solution can be easily obtained, since the eigenfunction have the following form [2]

$$
\phi_{\gamma}\left(q, q_{0}, Q_{T}=0\right)=\left(\frac{q^{2}}{q_{0}^{2}}\right)^{\gamma-1}
$$

Comparing Eq. (32) with Eq. (1) one can see that $q_{0} \sim 1 / R$, where $R$ denotes the size of the target. Repeating all the steps, that are given by Eq. (2) and Eq. (3), we obtain the solution in the form of Eq. (4); viz. 


$$
\begin{aligned}
\tilde{N}\left(q, Q_{T}=0, Y\right)= & \phi\left(\frac{1}{2}\right)\left(\frac{q_{0}^{2}}{q^{2}}\right)^{1 / 2} e^{\omega_{0} Y} \tilde{n}(q, Y) \\
& \text { where } \tilde{n}(q, Y)=\frac{1}{2 \sqrt{\pi D Y}} e^{-\frac{\tilde{\xi}^{2}}{4 D Y}}
\end{aligned}
$$

with $\tilde{\xi}=\ln \left(q^{2} / q_{0}^{2}\right)$. One can recognize that for the function $n$ we have the diffusion equation in the form:

$$
\frac{\partial}{\partial Y} \tilde{n}(\tilde{\xi}, Y)=D \frac{\partial^{2}}{\partial \tilde{\xi}^{2}} \tilde{n}(\tilde{\xi}, Y)
$$

Therefore, the BFKL equation describes that at each emission, $\ln \left(q^{2} / q^{2}\right)$ changes its value by a constant $\left(\ln \left(q^{2} / q^{\prime 2}\right)\right)^{2}=\delta$, and as a result after $n$ emissions we obtain $\ln ^{2}\left(p_{T}^{2} / q_{0}^{2}\right)=\delta n$. Since $n=\omega_{0} Y$ (see the previous section) we obtain $\left(\ln \left(q^{2} / q_{0}^{2}\right)\right)^{2} \delta \omega_{0} Y$. This estimate shows that $4 D=\delta \omega_{0}$. From this estimate we see that after $n$ emissions the typical transverse momenta increase as $\left\langle\left|p_{T}^{2}\right|\right\rangle=q_{0}^{2} \exp (\delta n)$, making the shift in $b\left\langle\left|\Delta b^{2}\right|\right\rangle_{n} \propto$ $1 /\left\langle\left|p_{T}^{2}\right|\right\rangle \sim\left(1 / q_{0}^{2}\right) \exp (-\delta n)$. Therefore, only a small number of steps at the beginning could participate in the increase of $b$ [see red lines in Fig. 1(b)].

\section{The Green function of the BFKL Pomeron} form:

The solution of Eq. (33) can be rewritten in the following

$$
\begin{aligned}
\tilde{N} & \left(q, Q_{T}=0, Y\right) \\
& =\int_{\epsilon-i \infty}^{\epsilon+i \infty} \frac{d \omega}{2 \pi i} \int_{\epsilon-i \infty}^{\epsilon+i \infty} \frac{d \gamma}{2 \pi i} \frac{1}{\omega-\omega(\gamma)} e^{\omega Y+(\gamma-1) \tilde{\xi}} \phi_{\text {int }}(\gamma)
\end{aligned}
$$

We introduce the Green function of the BFKL Pomeron as follows:

$$
G^{\mathrm{BFKL}}(\omega, \tilde{\xi})=\int_{\epsilon-i \infty}^{\epsilon+i \infty} \frac{d \gamma}{2 \pi i} \frac{1}{\omega-\omega(\gamma)} e^{\omega Y+(\gamma-1) \tilde{\xi}}
$$

The Green function in the $Y$ representation can be calculated as

$$
\begin{aligned}
G^{\mathrm{BFKL}}(Y, \tilde{\xi}) & =\frac{1}{q_{0}^{2}} \int_{\epsilon-i \infty}^{\epsilon+i \infty} \frac{d \omega}{2 \pi i} G^{\mathrm{BFKL}}\left(\omega, \tilde{\xi} ; Q_{T}=0\right) \\
& =\frac{1}{q_{0}^{2}} \int_{\epsilon-i \infty}^{\epsilon+i \infty} \frac{d \omega}{2 \pi i} \int_{\epsilon-i \infty}^{\epsilon+i \infty} \frac{d \gamma}{2 \pi i} \frac{1}{\omega-\omega(\gamma)} e^{\omega Y+(\gamma-1) \tilde{\xi}} \\
& \stackrel{\gamma=\frac{1}{2}+i \nu, \nu \ll 1}{\longrightarrow} \frac{1}{q q_{0}} e^{\omega_{0} Y} \int_{\epsilon-i \infty}^{\epsilon+i \infty} \frac{d \omega}{2 \pi i} \frac{1}{2 D \kappa_{0}} e^{-D \kappa_{0}^{2} Y+\kappa_{0} \tilde{\xi}} \\
& \text { where } \kappa_{0}=\sqrt{\frac{\left(\omega-\omega_{0}\right)}{D}}
\end{aligned}
$$

Integrating over $\kappa_{0}$ we obtain the solution of Eq. (33).
One can see that $G^{\mathrm{BFKL}}\left(Y=0, \tilde{\xi} ; Q_{T}=0\right)=\delta(\tilde{\xi})$ and therefore, the scattering amplitude can be found $\tilde{N}\left(Y ; q, Q_{T}=0\right)=\int d \tilde{\xi}_{0} G^{\mathrm{BFKL}}\left(\tilde{\xi}-\tilde{\xi}_{0}\right) \Phi_{\text {in }}\left(\tilde{\xi}_{0}\right)$, where $\Phi$ is the initial condition for the scattering amplitude. It should also be mentioned that factors $1 /\left(q q_{0}\right)$ are absorbed in the integration of $\tilde{\xi}$ in the diagrams for Pomeron interactions.

\section{The BFKL approach: Random walk in b}

As one can see from Eq. (33) we have introduced a new dimensional scale: $q_{0}$. It was introduced as the nonperturbative size of the target $q_{0} \sim 1 / R$, but its actual meaning is the separation scale: in perturbative QCD we can calculate only for $p_{T}>q_{0}$, while smaller transverse momenta have to be treated in nonperturbative approaches. From the solution of Eq. (33) one can see that the probability to have $p_{T}=q_{0}$ is small $\propto \frac{1}{2 \sqrt{D Y}}$ but not negligible. The gluons with transverse momenta of the order of $q_{0}$ could participate in the random walk in $b$, leading to $[5,6]$

$$
\left\langle\left|b^{2}\right|\right\rangle_{n}=\frac{1}{q_{0}^{2}} P_{q_{0}}^{n} n \propto \frac{1}{q_{0}^{2}} \frac{1}{\sqrt{n}} n \propto \frac{1}{q_{0}^{2}} \sqrt{Y}
$$

where $P_{q_{0}}^{n}$ denotes the probability to have the minimum momentum after $n$ emissions. In Refs. [5,6] the numerical coefficient in Eq. (38) is evaluated. Since the average $\left\langle\left|b^{2}\right|\right\rangle_{n}$ diverges at small $q$, in some sense the value of the coefficient is not important. However, we feel it is instructive to understand two qualitative features: the infrared divergency and the energy dependence.

First, we calculate the main ingredients of the calculation that we have discussed in Sec II A:

$$
\begin{aligned}
\left.\nabla_{Q_{T}} K_{\mathrm{em}}\left(\boldsymbol{q}-\boldsymbol{q}^{\prime}, \boldsymbol{Q}_{T}\right)\right|_{Q_{T}=0} & =-\frac{1}{\left(\boldsymbol{q}-\boldsymbol{q}^{\prime}\right)^{2}}\left(\frac{\boldsymbol{q}}{q^{2}}-\frac{\boldsymbol{q}^{\prime}}{q^{\prime 2}}\right) ; \\
\left.\nabla_{Q_{T}} K_{\mathrm{reg}}\left(\boldsymbol{q}-\boldsymbol{q}^{\prime}, \boldsymbol{Q}_{T}\right)\right|_{Q_{t}=0} & =-\frac{1}{\left(\boldsymbol{q}-\boldsymbol{q}^{\prime}\right)^{2}}\left(\frac{\boldsymbol{q}}{q^{\prime 2}}-\frac{\boldsymbol{q}^{\prime} q^{2}}{q^{\prime 4}}\right) ; \\
-\left.\nabla_{Q_{T}}^{2} K_{\mathrm{em}}\left(\boldsymbol{q}-\boldsymbol{q}^{\prime}, \boldsymbol{Q}_{T}\right)\right|_{Q_{T}=0} & =-\left.\Delta_{Q_{T}} K_{\mathrm{em}}\left(\boldsymbol{q}-\boldsymbol{q}^{\prime}, \boldsymbol{Q}_{T}\right)\right|_{Q_{T}=0} \\
& =\frac{2}{q^{2} q^{\prime 2}} ; \\
\left.\Delta_{Q_{T}} K_{\mathrm{reg}}\left(\boldsymbol{q}-\boldsymbol{q}^{\prime}, \boldsymbol{Q}_{T}\right)\right|_{Q_{T}=0} & =0
\end{aligned}
$$

As we have discussed in Sec. II A [see Eq. (23)], we can write the equation for $-\left.\nabla_{Q_{T}}^{2} \tilde{N}\left(Y ; q, Q_{T}\right)\right|_{Q_{T}=0}=$ $\int \frac{d^{2} b}{(2 \pi)^{2}} b^{2} \tilde{N}(q, b, Y)$ applying $-\nabla_{Q_{T}}^{2}$ to the both parts of Eq. (27). In doing so, we obtain: 


$$
\begin{aligned}
\frac{\partial}{\partial Y}\left(-\left.\nabla_{Q_{T}}^{2} \tilde{N}\left(Y ; q, Q_{T}\right)\right|_{Q_{T}=0}\right)= & \left.\left.\int d^{2} q^{\prime} \nabla_{Q_{T}}^{2} K\left(\boldsymbol{q}-\boldsymbol{q}^{\prime}, \boldsymbol{Q}_{T}\right)\right|_{Q_{T}=0} \tilde{N}\left(Y ; q^{\prime}, Q_{T}\right)\right|_{Q_{T}=0} \\
& -\left.2 \int d^{2} q^{\prime} \nabla_{Q_{T}} K\left(\boldsymbol{q}-\boldsymbol{q}^{\prime}, \boldsymbol{Q}_{T}\right)\right|_{Q_{T}=0} \cdot\left(\left.\nabla_{Q_{T}} \tilde{N}\left(Y ; q^{\prime}, Q_{T}\right)\right|_{Q_{T}=0}\right) \\
& +\left.\int d^{2} q^{\prime} K\left(\boldsymbol{q}-\boldsymbol{q}^{\prime}, \boldsymbol{Q}_{T}\right)\right|_{Q_{T}=0}\left(-\left.\nabla_{Q_{T}}^{2} \tilde{N}\left(Y ; q^{\prime}, Q_{T}\right)\right|_{Q_{T}=0}\right)
\end{aligned}
$$

To obtain the complete system of equations we need to add the equation for $\left.\nabla_{Q_{T}} \tilde{N}\left(Y ; q^{\prime}, Q_{T}\right)\right|_{Q_{T}=0}$. It takes the form:

$$
\begin{aligned}
\frac{\partial}{\partial Y}\left(\left.\nabla_{Q_{T}} \tilde{N}\left(Y ; q, Q_{T}\right)\right|_{Q_{T}=0}\right)= & \left.\left.\int d^{2} q^{\prime} \nabla_{Q_{T}} K\left(\boldsymbol{q}-\boldsymbol{q}^{\prime}, \boldsymbol{Q}_{T}\right)\right|_{Q_{T}=0} \tilde{N}\left(Y ; q^{\prime}, Q_{T}\right)\right|_{Q_{T}=0} \\
& +\left.\int d^{2} q^{\prime} K\left(\boldsymbol{q}-\boldsymbol{q}^{\prime}, \boldsymbol{Q}_{T}\right)\right|_{Q_{T}=0}\left(\left.\nabla_{Q_{T}} \tilde{N}\left(Y ; q^{\prime}, Q_{T}\right)\right|_{Q_{T}=0}\right) .
\end{aligned}
$$

Equation (41) can be rewritten as

$$
\left.\left(\frac{\partial}{\partial Y}-1\right) \nabla_{Q_{T}} \tilde{N}\left(Y ; q, Q_{T}\right)\right|_{Q_{T}=0}=\bar{\alpha}_{S} \frac{\boldsymbol{q}}{q^{2}}\left(\int_{q^{2}} \frac{d q^{\prime 2}}{q^{\prime 2}} \tilde{N}\left(Y ; q^{\prime}, Q_{T}=0\right)-\tilde{N}\left(Y ; q, Q_{T}=0\right)\right) .
$$

In the double Mellin transform of Eq. (36) the solution to Eq. (42) has the form:

$$
\left.\nabla_{Q_{T}} \tilde{N}\left(\omega ; \gamma, Q_{T}\right)\right|_{Q_{T}=0}=\bar{\alpha}_{S} \frac{\boldsymbol{q}}{q^{2}} \frac{1}{\omega-\omega(\gamma)}\left(\frac{1}{1-\gamma}-1\right)
$$

Plugging the solution of Eq. (43) into Eq. (40), we reduce this equation to the following one:

$$
\begin{aligned}
& \frac{\partial}{\partial Y}\left(-\left.\nabla_{Q_{T}}^{2} \tilde{N}\left(Y ; q, Q_{T}\right)\right|_{Q_{T}=0}\right)-\int d^{2} q^{\prime} K\left(\boldsymbol{q}-\boldsymbol{q}^{\prime}, \boldsymbol{Q}_{T}=0\right)\left(-\left.\nabla_{Q_{T}}^{2} \tilde{N}\left(Y ; q^{\prime}, Q_{T}\right)\right|_{Q_{T}=0}\right) \\
& \quad=2 \frac{\bar{\alpha}_{S}}{q^{2}}\left\{\int_{0}^{\infty} \frac{d q^{\prime 2}}{q^{\prime 2}} \tilde{N}\left(Y ; q^{\prime}, Q_{T}=0\right)+2 \bar{\alpha}_{S} \int_{0}^{q^{2}} \frac{d q^{\prime 2}}{q^{\prime 2}} \int_{\epsilon-i \infty}^{\epsilon+i \infty} \frac{d \gamma}{2 \pi i} \frac{1}{\omega-\omega(\gamma)}\left(\frac{1}{1-\gamma}-1\right) e^{\omega Y+(\gamma-1) \tilde{\xi}}\right\} .
\end{aligned}
$$

The main contributions to the integrals over $q^{\prime}$ stem from the region $q^{\prime} \rightarrow 0$. Taking this into account, the solution in the $\omega$-representation has the form:

$$
\left.\nabla_{Q_{T}}^{2} \tilde{N}\left(\omega ; q, Q_{T}\right)\right|_{Q_{T}=0}=2 \frac{\bar{\alpha}_{S}}{q^{2}} \frac{1+\bar{\alpha}_{S}}{D^{2} \kappa_{0}^{2}} \propto \frac{1}{\omega-\omega_{0}} .
$$

Therefore, we see from Eq. (45) that $\left.\nabla_{Q_{T}}^{2} \tilde{N}\left(\omega ; q, Q_{T}\right)\right|_{Q_{T}=0} \propto$ $e^{\omega_{0} Y}$.

We need to divide this solution by the $\left.\tilde{N}\left(Y ; q^{\prime}, Q_{T}\right)\right|_{Q_{T}=0}$ and finally, we obtain for $\xi^{2} \ll D Y$ that

$$
\left\langle b^{2}\right\rangle=2 \bar{\alpha}_{S}\left(1+\bar{\alpha}_{S}\right) \sqrt{\frac{\pi}{D}} \frac{1}{q^{2}} \sqrt{Y}=\alpha_{\mathrm{eff}}^{\prime} \sqrt{Y} .
$$

As was shown in Refs. [5,6], the second term does not have the suppression of the order of $\bar{\alpha}_{S}$, as we can see from Eq. (45). Such an enhancement comes from the replacement in Eq. (40)

$$
\begin{aligned}
& \left.2 \int d^{2} q^{\prime} \nabla_{Q_{T}} K\left(\boldsymbol{q}-\boldsymbol{q}^{\prime}, \boldsymbol{Q}_{T}\right)\right|_{Q_{T}=0} \cdot\left(\left.\nabla_{Q_{T}} \tilde{N}\left(Y ; q^{\prime}, Q_{T}\right)\right|_{Q_{T}=0}\right) \\
& \left.\rightarrow 2 \int d^{2} q^{\prime} \nabla_{Q_{T}} K\left(\boldsymbol{q}-\boldsymbol{q}^{\prime}, \boldsymbol{Q}_{T}\right)\right|_{Q_{T}=0} \otimes \phi_{1}\left(Y-Y^{\prime}, q^{\prime}, q^{\prime}, \theta\right) \\
& \otimes\left(\left.\nabla_{Q_{T}} \tilde{N}\left(Y^{\prime} ; q^{\prime \prime}, Q_{T}\right)\right|_{Q_{T}=0}\right)
\end{aligned}
$$

$\otimes$ stands for all the required integrations. The eigenfunction $\phi_{1}$ [3] depends on the angles between $\boldsymbol{q}^{\prime}$ and $\boldsymbol{q}^{\prime}$ and has an intercept which is negative and $\propto \bar{\alpha}_{S}$. Integration over $Y^{\prime}$ leads to the factor $1 / \bar{\alpha}_{S}$ which compensates the small value of $\bar{\alpha}_{S}$. This contribution actually leads to the change of the numerical coefficient, which does not have much meaning, since $\left\langle b^{2}\right\rangle$ is infrared unstable, and $q \rightarrow q_{0}$ gives the main contribution. The value of the scale $q_{0}$ is not determined in our approach. Hence, we introduce a new dimensional scale $\alpha_{\text {eff }}^{\prime}$ which, we hope, will heal the large $b$ behavior of the scattering amplitude. 


\section{A NEW NONLINEAR EVOLUTION EQUATION}

In this section we propose a nonlinear equation, which takes into account the random walk both in $\ln \left(p_{T}\right)$, and in $b$, and which will transform the CGC/saturation approach into an effective theory, describing QCD at high energies. The effort to find such an equation covers a long span in time, from the first GLR equation [37](see also Refs. [36,38,39]) till recent papers. Our goal is to introduce the random walk in $b$ in the framework of the nonlinear evolution equations, which guarantee the unitarity of the scattering amplitude. The CGC/saturation approach is based on the nonlinear Balitsky -Kovchegov equation [40], which itself has problems with the behavior of the scattering amplitude at large $b$, typical for perturbative QCD.

We proposed the following generalization of the Balitsky-Kovchegov nonlinear evolution equation [40]:

$$
\begin{aligned}
\frac{\partial}{\partial Y} N(\boldsymbol{r}, \boldsymbol{b}, Y)= & \bar{\alpha}_{S} \int \frac{d^{2} \boldsymbol{r}^{\prime}}{2 \pi} K\left(\boldsymbol{r}^{\prime}, \boldsymbol{r}-\boldsymbol{r}^{\prime} ; \boldsymbol{r}\right)\left\{N\left(\boldsymbol{r}^{\prime}, \boldsymbol{b}-\frac{1}{2}\left(\boldsymbol{r}-\boldsymbol{r}^{\prime}\right), Y\right)+N\left(\boldsymbol{r}-\boldsymbol{r}^{\prime}, \boldsymbol{b}-\frac{1}{2} \boldsymbol{r}^{\prime}, Y\right)-N(\boldsymbol{r}, \boldsymbol{b}, Y)\right. \\
& \left.-N\left(\boldsymbol{r}-\boldsymbol{r}^{\prime}, \boldsymbol{b}-\frac{1}{2} \boldsymbol{r}^{\prime}, Y\right) N\left(\boldsymbol{r}^{\prime}, \boldsymbol{b}-\frac{1}{2}\left(\boldsymbol{r}-\boldsymbol{r}^{\prime}\right), Y\right)\right\}+\left(\alpha_{\mathrm{eff}}^{\prime} \nabla_{b}^{2}\right)^{2} N(\boldsymbol{r}, \boldsymbol{b}, Y) \\
\stackrel{b \gg r, r^{\prime}}{\longrightarrow} & \bar{\alpha}_{S} \int \frac{d^{2} \boldsymbol{r}^{\prime}}{2 \pi} K\left(\boldsymbol{r}^{\prime}, \boldsymbol{r}-\boldsymbol{r}^{\prime} ; \boldsymbol{r}\right)\left\{N\left(\boldsymbol{r}^{\prime}, \boldsymbol{b}, Y\right)+N\left(\boldsymbol{r}-\boldsymbol{r}^{\prime}, \boldsymbol{b}, Y\right)-N(\boldsymbol{r}, \boldsymbol{b}, Y)-N\left(\boldsymbol{r}-\boldsymbol{r}^{\prime}, \boldsymbol{b}, Y\right) N\left(\boldsymbol{r}^{\prime}, \boldsymbol{b}, Y\right)\right\} \\
& +\left(\alpha_{\mathrm{eff}}^{\prime} \nabla_{b}^{2}\right)^{2} N(\boldsymbol{r}, \boldsymbol{b}, Y),
\end{aligned}
$$

where $\alpha_{\text {eff }}^{\prime}$ is a new dimensional scale. We believe that Eq. (48) is the correct way to introduce this scale for the nonlinear evolution.

\section{A. The BFKL Pomeron with diffusion in $b$}

First, we solve the new equation at large $b$, where only the linear part contributes. The equation takes the form:

$$
\begin{aligned}
\frac{\partial}{\partial Y} N(\boldsymbol{r}, \boldsymbol{b}, Y)= & \bar{\alpha}_{S} \int \frac{d^{2} \boldsymbol{r}^{\prime}}{2 \pi} K\left(\boldsymbol{r}^{\prime}, \boldsymbol{r}-\boldsymbol{r}^{\prime} ; \boldsymbol{r}\right)\left\{N\left(\boldsymbol{r}^{\prime}, \boldsymbol{b}, Y\right)\right. \\
& \left.+N\left(\boldsymbol{r}-\boldsymbol{r}^{\prime}, \boldsymbol{b}, Y\right)-N(\boldsymbol{r}, \boldsymbol{b}, Y)\right\} \\
& +\left(\alpha_{\mathrm{eff}}^{\prime} \nabla_{b}^{2}\right)^{2} N(\boldsymbol{r}, \boldsymbol{b}, Y) .
\end{aligned}
$$

We can obtain the solution to this equation considering it in the form: $N(\boldsymbol{r}, \boldsymbol{b}, Y)=G(\boldsymbol{r}, Y) \Phi(Y ; b)$ where $G(\boldsymbol{r}, Y)$ is the Green's function of the BFKL equation and $\Phi(Y ; b)$ satisfies the following equation

$$
\frac{\partial}{\partial Y} \Phi(\boldsymbol{b}, Y)=\left(\alpha_{\mathrm{eff}}^{\prime} \nabla_{b}^{2}\right)^{2} \Phi(\boldsymbol{b}, Y) .
$$

In $\omega$-representation:

$$
\Phi(\boldsymbol{b}, Y)=\int_{\epsilon-i \infty}^{\epsilon+i \infty} \frac{d \omega}{2 \pi i} e^{\omega Y} \Phi(\boldsymbol{b}, \omega) .
$$

Equation (49) takes the form

$$
\left(\alpha_{\mathrm{eff}}^{\prime} \nabla_{b}^{2}\right)^{2} \Phi(\boldsymbol{b}, \omega)=\omega \Phi(\boldsymbol{b}, \omega)
$$

The general solution to Eq. (51) can be written (see Ref. [41] formula 9.4.3) as

$$
\Phi(\boldsymbol{b}, \omega)=\Phi_{-}(\boldsymbol{b}, \omega)+\Phi_{+}(\boldsymbol{b}, \omega)
$$

where $\Phi_{ \pm}$are the solution to the following equations:

$$
\alpha_{\mathrm{eff}}^{\prime} \nabla_{b}^{2} \Phi_{ \pm}(\boldsymbol{b}, \omega) \pm \sqrt{\omega} \Phi_{ \pm}(\boldsymbol{b}, \omega)=0 .
$$

We restrict ourself by the solution $\Phi(\boldsymbol{b}, \omega)=\Phi_{+}(\boldsymbol{b}, \omega)$ for which the Green's function is equal to

$$
\Phi_{+}(\boldsymbol{b}, \omega)=\int \frac{d^{2} Q_{T}}{(2 \pi)^{2}} e^{i \boldsymbol{Q}_{T} \cdot \boldsymbol{b}} \frac{\phi_{+}^{\mathrm{in}}(\omega)}{\alpha_{\mathrm{eff}}^{\prime} Q_{T}^{2}+\sqrt{\omega}}
$$

where $\phi_{+}^{\text {in }}(\omega)$ should be found from the initial condition at $Y=0$. Searching for the Green's function of Eq. (54) we impose the initial condition:

$$
\Phi_{+}(\boldsymbol{b}, Y=0)=\delta^{(2)}(\boldsymbol{b})
$$

which results in $\phi_{+}^{\text {in }}(\omega)=1 /(2 \sqrt{\omega})$.

Equation (54) has the same form as the Green function of the modified BFKL Pomeron, suggested in Ref. [23] (see also the review section in Ref. [28]). In these papers a theoretical approach is proposed, which is based on the assumption, that the BFKL Pomeron, being perturbative in nature, takes into account rather short distances (say of the order of $1 / m_{G}$, where $m_{G}$ denotes the mass of the lightest glueball); and the long distance contribution can be described by the exchange of pions $\left(1 / 2 \mu \gg 1 / m_{G}\right.$, where $\mu$ is the pion mass). The calculations are based on the $t$-channel unitarity, as was proposed in Ref. [42], and on the finding, that the intercept of the BFKL Pomeron does not depend on the model of the long distances contribution [22]. 
Taking the integral over $d^{2} Q_{T}$ we obtain:

$$
\Phi_{+}(\boldsymbol{b}, \omega)=\frac{1}{4 \pi \alpha_{\mathrm{eff}}^{\prime} \sqrt{\omega}} K_{0}\left(\frac{b}{\sqrt{\alpha_{\mathrm{eff}}^{\prime}}} \omega^{1 / 4}\right)
$$

and for $\Phi(\boldsymbol{b}, Y)$ we have

$$
\Phi(\boldsymbol{b}, Y)=\int_{\epsilon-i \infty}^{\epsilon+i \infty} \frac{d \omega}{8 \pi^{2} i} \frac{1}{\alpha_{\mathrm{eff}}^{\prime} \sqrt{\omega}} e^{\omega Y} K_{0}\left(\frac{b}{\sqrt{\alpha_{\mathrm{eff}}^{\prime}}} \omega^{1 / 4}\right) .
$$

For large $b$ we can take the integral over $\omega$, plugging the asymptotic behavior of the $K_{0}$ function, and using the method of steepest descend. Eq. (57) takes the form:

$$
\begin{aligned}
\Phi(\boldsymbol{b}, Y)= & \int_{\epsilon-i \infty}^{\epsilon+i \infty} \frac{d \omega}{8 \pi^{2} i} \frac{1}{\alpha_{\mathrm{eff}}^{\prime} \sqrt{\omega}} \\
& \times e^{\omega Y} \sqrt{\frac{\pi}{2}}\left(\frac{1}{\omega}\right)^{1 / 8} \frac{1}{\sqrt{\frac{b}{\sqrt{\alpha_{\mathrm{eff}}^{\prime}}}}} e^{-\frac{b}{\sqrt{\alpha_{\mathrm{eff}}^{\prime}}} \omega^{1 / 4}} .
\end{aligned}
$$

The equation for the saddle point has the following form

$$
Y-\frac{1}{4} \frac{\frac{b}{\sqrt{\alpha_{\mathrm{eff}}^{\prime}}}}{\omega_{\mathrm{SP}}^{3 / 4}}=0 ; \quad \omega_{\mathrm{SP}}=\left(\frac{b}{4 Y \sqrt{\alpha_{\mathrm{eff}}^{\prime}}}\right)^{4 / 3} .
$$

From Eq. (59) we obtain for the integral over $\omega$ :

$$
\Phi(\boldsymbol{b}, Y)=\frac{4 \pi}{\sqrt{6} \alpha_{\mathrm{eff}}^{\prime}}\left(\frac{\alpha_{\mathrm{eff}}^{\prime}}{4 Y b^{2}}\right)^{1 / 3} \exp \left(-\frac{3}{4} \mathcal{Z}\right)
$$

where

$$
\mathcal{Z}=\left(\frac{b^{4}}{4 \alpha_{\mathrm{eff}}^{\prime 2} Y}\right)^{1 / 3}
$$

Finally, from $N(\boldsymbol{r}, \boldsymbol{b}, Y)=G(\boldsymbol{r}, Y) \Phi(Y ; b)$ we obtain

$$
\begin{aligned}
G(\boldsymbol{r}, \boldsymbol{b}, Y) & =\int_{\epsilon-i \infty}^{\epsilon+i \infty} \frac{d \gamma}{2 \pi i} e^{\bar{\alpha}_{S} \chi(\gamma) Y-(-\gamma) \xi} \Phi(Y ; b) \\
& =\frac{r}{R} e^{\omega_{0} Y} \frac{1}{2 \sqrt{\pi D Y}} e^{-\frac{\xi^{2}}{4 D Y}} \Phi(Y ; b)
\end{aligned}
$$

and $\Phi(Y ; b)$ is given by Eq. (60). In Eq. (62) we integrated over $\gamma$ using the diffusion expansion for the kernel, $\chi(\gamma)$ for $\gamma \rightarrow \frac{1}{2}$.

For the region of not large $b$ we obtain the solution which has a cumbersome form:

$$
\begin{aligned}
& \frac{1}{16 \alpha_{\mathrm{eff}}^{\prime 2} Y}\left(-4 \sqrt{\pi} \sqrt{-\alpha_{\mathrm{eff}}^{\prime 2} Y_{0}} F_{2}\left(; \frac{1}{2}, 1 ;-\frac{b^{4}}{256 \alpha_{\mathrm{eff}}^{\prime 2} Y}\right)\right. \\
& \left.-b_{0}^{2} F_{2}\left(; \frac{3}{2}, \frac{3}{2} ;-\frac{b^{4}}{256 \alpha_{\mathrm{eff}}^{\prime 2} Y}\right)\right)
\end{aligned}
$$

where ${ }_{0} F_{2}$ is the hyperbolic function (see formula $\mathbf{9 . 1 4}$ of Ref. [43]).

As we have mentioned above the solution of Eq. (61) is the Green's function of Eq. (50), and it preserves the remarkable property of the Green's function of the BFKL Pomeron:

$$
\begin{aligned}
\Phi(\boldsymbol{b}, Y)= & \int d^{2} b^{\prime} \Phi\left(\boldsymbol{b}-\boldsymbol{b}^{\prime}, Y-Y^{\prime}\right) \Phi\left(\boldsymbol{b}^{\prime}, Y^{\prime}\right) \\
= & \int d^{2} b^{\prime} \int \frac{d^{2} Q_{T}^{\prime}}{4 \pi^{2}} \exp \left(\alpha_{\mathrm{eff}}^{\prime 2} Q_{T}^{\prime 4} Y^{\prime}+i \boldsymbol{q}_{T}^{\prime} \cdot \boldsymbol{b}^{\prime}\right) \\
& \times \int \frac{d^{2} Q_{T}}{4 \pi^{2}} \exp \left(\alpha_{\mathrm{eff}}^{\prime 2} Q_{T}^{4}\left(Y-Y^{\prime}\right)+i \boldsymbol{Q}_{T} \cdot\left(\boldsymbol{b}-\boldsymbol{b}^{\prime}\right)\right) \\
= & \int d^{2} Q_{T} \exp \left(\alpha_{\mathrm{eff}}^{\prime 2} Q_{T}^{\prime 4}+i \boldsymbol{q}_{T}^{\prime} \cdot \boldsymbol{b}\right) .
\end{aligned}
$$

In Eq. (64) we use Eq. (54) for $\Phi_{+}(\boldsymbol{b}, \omega)$ which leads to the following representation for $\Phi(\boldsymbol{b}, Y)$

$$
\begin{aligned}
\Phi(\boldsymbol{b}, Y) & =\int_{\epsilon-i \infty}^{\epsilon+i \infty} \frac{d \omega}{2 \pi i} e^{\omega Y} \Phi_{+}(\boldsymbol{b}, \omega) \\
& =\int d^{2} Q_{T} \exp \left(\alpha_{\mathrm{eff}}^{\prime 2} Q_{T}^{\prime 4}+i \boldsymbol{q}_{T}^{\prime} \cdot \boldsymbol{b}\right) .
\end{aligned}
$$

The integration over $d^{2} b^{\prime}$ in Eq. (64) generates $\delta^{(2)}\left(\boldsymbol{Q}_{T}-\boldsymbol{Q}_{T}^{\prime}\right)$, which results in Eq. (64).

Using Eq. (64) we can prove that for the Green's function of Eq. (62) we have the following equation:

$$
\begin{aligned}
G(\boldsymbol{r}, \boldsymbol{b}, \boldsymbol{R}, Y)= & \int d^{2} r^{\prime} d^{2} b^{\prime} G\left(\boldsymbol{r}, \boldsymbol{b}-\boldsymbol{b}^{\prime}, \boldsymbol{r}^{\prime}, Y-Y^{\prime}\right) G\left(\boldsymbol{r}^{\prime}, \boldsymbol{b}^{\prime}, \boldsymbol{R}, Y^{\prime}\right) \\
= & \int d \xi^{\prime} d^{2} b^{\prime} \int \frac{d \gamma^{\prime}}{2 \pi i} \int \frac{d^{2} Q_{T}^{\prime}}{4 \pi^{2}} \exp \left(\left(\bar{\alpha}_{S} \chi\left(\gamma^{\prime}\right)+\alpha_{\mathrm{eff}}^{\prime 2} Q_{T}^{\prime 4}\right) Y^{\prime}+\left(\gamma^{\prime}-1\right) \xi^{\prime}+i \boldsymbol{q}_{T}^{\prime} \cdot \boldsymbol{b}^{\prime}\right) \\
& \times \int \frac{d \gamma}{2 \pi i} \int \frac{d^{2} Q_{T}}{4 \pi^{2}} \exp \left(\left(\bar{\alpha}_{S} \chi(\gamma)+\alpha_{\mathrm{eff}}^{\prime 2} Q_{T}^{4}\right)\left(Y-Y^{\prime}\right)+(\gamma-1)\left(\xi-\xi^{\prime}\right)+i \boldsymbol{Q}_{T} \cdot\left(\boldsymbol{b}-\boldsymbol{b}^{\prime}\right)\right)
\end{aligned}
$$

where $\xi^{\prime}=\ln \left(r^{\prime 2} / R^{2}\right)$. 
The integration over $\xi^{\prime}$ and $\boldsymbol{b}^{\prime}$ gives $\delta\left(\gamma-\gamma^{\prime}\right)$ and $\delta^{(2)}\left(\boldsymbol{Q}_{T}-\boldsymbol{Q}_{T}^{\prime}\right)$, which leads to the equation Eq. (67).

One can see that Eq. (62) has the same main features as the Green function of the modified BFKL Pomeron, in which the contribution of long distances stems from the exchange of pions [23].

In particular, the unitarization leads to the Froissart disc with radius

$$
\frac{R}{\sqrt{\alpha_{\text {eff }}^{\prime}}}=\frac{4}{3^{3 / 4}} \omega_{0}^{3 / 4} Y-\frac{3^{1 / 4}}{4 D \omega_{0}^{1 / 4}} \frac{\xi^{2}}{Y}
$$

whose edge decreases with $b$ as $e^{-\frac{3}{4}\left(\frac{b^{4}}{4 \alpha^{2}} \text { eff }^{1}\right)^{1 / 3}}$ which corresponds to the diffusion in $b$ with $\left\langle b^{2}\right\rangle \propto \sqrt{Y}$, as follows from Sec. II B 4.

\section{B. Solution in the vicinity of the saturation scale}

The behavior of the scattering dipole amplitude in the vicinity of the saturation scale can be found from the solution of the linear equation $[37,44]$. We will use the solution to Eq. (48) in the form of Eq. (60), and will take the integral over $\gamma$ using the method of steepest descend. The equation for the saddle point in $\gamma$ has the form:

$$
\left.\bar{\alpha}_{S} \frac{d \chi(\gamma)}{d \gamma}\right|_{\gamma=\gamma_{\mathrm{SP}}} Y+\xi=0
$$

The second condition is that the scattering amplitude is a constant for $r^{2} Q_{s}^{2}=1$, which has the form

$$
\bar{\alpha}_{S} \chi\left(\gamma_{\mathrm{SP}}\right) Y+\left(\gamma_{\mathrm{SP}}-1\right) \xi-\frac{3}{4} \mathcal{Z}=0 .
$$

Plugging Eq. (69) into Eq. (70) we obtain the following equation for $\gamma_{\mathrm{SP}}$.

$$
\bar{\alpha}_{S}\left(\chi\left(\gamma_{\mathrm{SP}}\right)+\left.\left(1-\gamma_{\mathrm{SP}}\right) \frac{d \chi(\gamma)}{d \gamma}\right|_{\gamma=\gamma_{\mathrm{SP}}}\right) Y=\frac{3}{4} \mathcal{Z} .
$$

For $\mathcal{Z}=0$ the solution to Eq. (71) is $\gamma_{\mathrm{SP}}=\gamma_{c r} \approx 0.37$. If $\mathcal{Z} \ll \bar{\alpha}_{S} Y$ we can find the solution to Eq. (71) assuming, that $\gamma_{\mathrm{SP}}=\gamma_{c r}+\delta \gamma$ with $\delta \gamma \ll \gamma_{c r}$. The value of $\delta \gamma$ from Eq. (71) turns out to be equal to

$$
\delta \gamma=\frac{3}{4} \frac{1}{\left.\left(1-\gamma_{c r}\right) \frac{d \chi(\gamma)}{d \gamma}\right|_{\gamma=\gamma_{c r}}} \frac{\mathcal{Z}}{\bar{\alpha}_{S} Y} .
$$

Substituting $\gamma_{\mathrm{SP}}=\gamma_{c r}+\delta \gamma$ into Eq. (69), we obtain the expression for the saturation momentum:

$$
\xi_{s}=\ln \left(Q_{s}^{2}\right)=\bar{\alpha}_{S} \frac{\chi\left(\gamma_{c r}\right)}{1-\gamma_{c r}} Y-\frac{3}{4\left(1-\gamma_{c r}\right)} \mathcal{Z}
$$

In the vicinity of the saturation scale the scattering amplitude shows geometric scaling behavior [45-48] and has the following form [44] for $\tau \sim 1$ :

$$
N(r, b, Y)=N_{0}\left(r^{2} Q_{s}^{2}(Y, b)\right)^{1-\gamma_{c r}}=N_{0} \tau^{1-\gamma_{c r}}
$$

where $N_{0}$ is a constant and we assumed that $\delta \gamma \ln \tau \ll 1$.

Using Eq. (73) for $Q_{s}$ we see that $N(r, b, Y)$ of Eq. (74) can be rewritten in the form:

$N(r, b, Y)=N_{0}\left(r^{2} Q_{S}^{2}(Y, b=0)\right)^{1-\gamma_{c r}} e^{-\frac{3}{4}\left(\frac{b^{4}}{\left.4 \alpha^{2}{ }_{\text {eff }}\right)^{1 / 3}}\right.}$

for the kinematic region

$$
r^{2} Q_{s}^{2}(Y=0) e^{\bar{\alpha}_{S} \frac{\gamma(\gamma c r)}{1-\gamma_{c r}} Y-\frac{3}{4\left(1-\gamma_{c r}\right)} \mathcal{Z}} \sim 1 .
$$

\section{The scattering amplitude in the deep saturation region}

We use the approach of Ref. [46] for linearizing Eq. (48) deep inside the saturation region $\left(r^{2} Q_{s}^{2}(Y, b) \gg 1\right)$ : we assume that $N(r, b, Y) \rightarrow 1$ and neglect the contributions, which are proportional to $\Delta^{2}(r, b, Y) \ll 1$ where $N(r, b, Y)=$ $1-\Delta(r, b, Y)$. Eq. (48) reduces to the following linear equation for $\Delta(r, b, Y)$

$$
\begin{aligned}
\frac{\partial}{\partial Y} \Delta(\boldsymbol{r}, \boldsymbol{b}, Y)= & -\bar{\alpha}_{S} \int_{1 / Q_{s}(Y, b)}^{r} \frac{d^{2} \boldsymbol{r}^{\prime}}{2 \pi} K\left(\boldsymbol{r}^{\prime}, \boldsymbol{r}-\boldsymbol{r}^{\prime} ; \boldsymbol{r}\right) \Delta(\boldsymbol{r}, \boldsymbol{b}, Y) \\
& +\left(\alpha_{\mathrm{eff}}^{\prime} \nabla_{b}^{2}\right)^{2} \Delta(\boldsymbol{r}, \boldsymbol{b}, Y) .
\end{aligned}
$$

In the previous section we found, that the scattering amplitude for $\tau \sim 1$ shows geometric scaling behavior, being a function of only one variable $\tau$. The first term in the r.h.s. of Eq. (77) is equal to $-\bar{\alpha}_{S} \ln \tau \equiv-\bar{\alpha}_{S} \zeta$. We can re-write $\frac{\partial}{\partial Y}$ as

$\frac{\partial}{\partial Y}=\frac{\partial}{\partial \zeta} \frac{\partial \zeta}{\partial Y}=\left(\bar{\alpha}_{S} \frac{\chi\left(\gamma_{c r}\right)}{1-\gamma_{c r}}+\frac{1}{4\left(1-\gamma_{c r}\right) Y} \mathcal{Z}\right) \frac{\partial}{\partial \zeta}$.

Assuming that $\frac{1}{4\left(1-\gamma_{c r}\right) Y} \mathcal{Z} \ll 1$ we can neglect this contribution and rewrite Eq. (77) in the form

$$
\begin{aligned}
& \bar{\alpha}_{S} \frac{\chi\left(\gamma_{c r}\right)}{1-\gamma_{c r}} \frac{\partial \Delta(\zeta, b)}{\partial \zeta} \\
& \quad=-\bar{\alpha}_{S} \zeta \Delta(\zeta, b)+\left(\alpha_{\mathrm{eff}}^{\prime} \nabla_{b}^{2}\right)^{2} \Delta(\zeta, b)
\end{aligned}
$$

where

$$
\zeta=\xi+\xi_{s}=\xi+\bar{\alpha}_{S} \frac{\chi\left(\gamma_{c r}\right)}{1-\gamma_{c r}} Y-\frac{3}{4\left(1-\gamma_{c r}\right)} \mathcal{Z}
$$

The solution to Eq. (79) can be written in the form (see Sec. III A):

$\Delta(\zeta, b)=\exp \left(-\frac{\zeta^{2}}{2 \kappa}\right) \Phi(\zeta, b) \quad$ with $\quad \kappa=\frac{\chi\left(\gamma_{c r}\right)}{1-\gamma_{c r}}$ 
This solution violates the geometric scaling behavior of the scattering amplitude, leading to the additional suppression of the scattering amplitude at large $b$. However, it should be emphasized, that the main dependence on the impact parameter is included in the $b$ dependence of the variable $\zeta$ [see Eq. (80)]. For the BK equation the asymptotic behavior of the scattering amplitude is determined by $\Delta(\zeta, b)=$ Const $\exp \left(-\frac{\zeta^{2}}{2 \kappa}\right)$, with a constant which we cannot estimate. The solution of Eq. (81) states that instead of Const we can have a function $\Phi(Y, b)$. However, in the next section we will argue that actually we can replace $\Phi(Y, b)$ by 1 .

\section{Instructive example: Solution to leading twist nonlinear equation}

The general nonlinear evolution that is given by Eq. (48) is difficult to analyze analytically for the full BFKL kernel of Eq. (30) or Eq. (3). This kernel includes the summation over all twist contributions. We would like to start with a simplified version of the kernel in which we restrict ourselves to the leading twist term only, which has the form

$\chi(\gamma)=\left\{\begin{array}{l}\frac{1}{\gamma} \quad \text { for } \tau=r Q_{s}<1 \operatorname{summing}\left(\ln \left(1 /\left(r \Lambda_{\mathrm{QCD}}\right)\right)\right)^{n} ; \\ \frac{1}{1-\gamma} \quad \text { for } \tau=r Q_{s}>1 \operatorname{summing}\left(\ln \left(r Q_{s}\right)\right)^{n} ;\end{array}\right.$

instead of the full expression of Eq. (30).

As indicated in Eq. (82) we have two types of logs: $\left(\bar{\alpha}_{S} \ln \left(r \Lambda_{\mathrm{QCD}}\right)\right)^{n}$ in the perturbative QCD kinematic region where $r Q_{s}(Y, b) \equiv \tau \ll 1$; and $\left(\bar{\alpha}_{S} \ln \left(r Q_{s}(Y, b)\right)\right)^{n}$ inside the saturation domain $(\tau \gg 1)$, where $Q_{s}(Y, b)$ denotes the saturation scale. To sum these logs it is necessary to modify the BFKL kernel in different ways, in the two kinematic regions, as shown in Eq. (82).

Inside the saturation region where $\tau=r^{2} Q_{s}^{2}(Y, b)>1$ the $\operatorname{logs}$ originate from the decay of a large size dipole into one small size dipole and one large size dipole. However, the size of the small dipole is still larger than $1 / Q_{s}$. This observation can be translated in the following form of the kernel

$$
\begin{aligned}
\int & K\left(\boldsymbol{x}_{01} ; \boldsymbol{x}_{02}, \boldsymbol{x}_{12}\right) d^{2} x_{02} \\
& \rightarrow \pi \int_{1 / Q_{s}^{2}(Y, b)}^{x_{01}^{2}} \frac{d x_{02}^{2}}{x_{02}^{2}}+\pi \int_{1 / Q_{s}^{2}(Y, b)}^{x_{01}^{2}} \frac{d\left|\boldsymbol{x}_{01}-\boldsymbol{x}_{02}\right|^{2}}{\left|\boldsymbol{x}_{01}-\boldsymbol{x}_{02}\right|^{2}}
\end{aligned}
$$

Inside the saturation region Eq. (48) takes the form

$$
\begin{aligned}
\frac{\partial^{2} \tilde{N}(Y ; \xi, \boldsymbol{b})}{\partial Y \partial \xi}= & \bar{\alpha}_{S}\left\{\left(1-\frac{\partial \tilde{N}(Y ; \boldsymbol{r}, \boldsymbol{b})}{\partial \xi}\right) \tilde{N}(Y ; \boldsymbol{\xi}, \boldsymbol{b})\right\} \\
& +\left(\alpha_{\mathrm{eff}}^{\prime} \nabla_{b}^{2}\right)^{2} \frac{\partial \tilde{N}(Y ; \boldsymbol{\xi}, \boldsymbol{b})}{\partial \xi}
\end{aligned}
$$

where $\tilde{N}(Y ; \xi, \boldsymbol{b})=\int r^{2} d r^{\prime 2} N\left(Y ; \boldsymbol{r}^{\prime}, \boldsymbol{b}\right) / r^{\prime 2}=\int^{\xi} d \xi^{\prime} N\left(Y, \xi^{\prime}, \boldsymbol{b}\right)$.
The advantage of the simplified kernel of Eq. (82) is that, in the double $\log$ approximation (DLA) for $\tau<1$, it provides a matching with the DGLAP evolution equation [49].

Introducing

$$
\frac{\partial \tilde{N}(Y ; \boldsymbol{\xi}, \boldsymbol{b})}{\partial \xi}=1-e^{-\phi(Y, \xi, \boldsymbol{b})}
$$

we can rewrite Eq. (84) in the form:

$$
\begin{aligned}
\frac{\partial \phi(Y, \xi, \boldsymbol{b})}{\partial Y} e^{-\phi(Y, \xi, \boldsymbol{b})}= & \bar{\alpha}_{S} e^{-\phi(Y, \xi, \boldsymbol{b})} \tilde{N}(Y ; \xi, \boldsymbol{b}) \\
& +\left(\alpha_{\mathrm{eff}}^{\prime} \nabla_{b}^{2}\right)^{2} e^{-\phi(Y, \xi, \boldsymbol{b})}
\end{aligned}
$$

Assuming that $\phi(\zeta, b)=\phi(Y, \xi)+\tilde{\phi}(Y, b)$ we can rewrite Eq. (86) as follows:

$\frac{\partial \phi(Y, \boldsymbol{\xi}, \boldsymbol{b})}{\partial Y}=\bar{\alpha}_{S} \tilde{N}(Y ; \boldsymbol{\xi}, \boldsymbol{b})+\underbrace{\left(e^{\tilde{\phi}(Y, \boldsymbol{b})}\left(\alpha_{\mathrm{eff}}^{\prime} \nabla_{b}^{2}\right)^{2} e^{-\tilde{\phi}(Y, \boldsymbol{b})}\right)}_{\text {function of } Y, b}$.

Using Eq. (78) and Eq. (80) with

$$
\zeta=\xi+\xi_{s}=\xi+4 \bar{\alpha}_{S} Y-\frac{3}{2} \mathcal{Z} .
$$

We can reduce Eq. (87), applying $\frac{\partial}{\partial \xi}$ to the both sides of this equation, to the following equation

$$
\frac{d^{2} \phi(\zeta)}{d \zeta^{2}}=\frac{1}{4}\left(1-e^{-\phi(\zeta)}\right)
$$

Introducing $\frac{d \phi(\zeta)}{d \zeta}=F(\phi)$ we can rewrite Eq. (89) in the form

$$
\begin{aligned}
\frac{1}{2} \frac{d F^{2}(\phi)}{d \phi} & =\frac{1}{4}\left(1-e^{-\phi}\right) ; \\
F^{2}(\phi) & =\int d \phi \frac{1}{2}\left(1-e^{-\phi}\right)=\frac{1}{2}\left(-1+\phi+e^{-\phi}\right)+C \mid(Y, b) .
\end{aligned}
$$

Finally,

$$
\sqrt{2} \int_{\phi_{0}}^{\phi} \frac{d \phi^{\prime}}{\sqrt{-1+\phi^{\prime}+e^{-\phi^{\prime}}+C(Y, b)}}=\zeta+\text { Const. }
$$

Further information regarding this equation can be found in the book of Ref. [41] (see formula 4.1.1.). $\phi_{0}$ is the value of $\phi$ at $\zeta=0$. One can see that if $\phi_{0} \ll 1$ we have $\phi=\phi_{0} e^{\frac{1}{2} \zeta}$ for $\zeta \rightarrow 0$ for Const $=0$ and the arbitrary function $C(Y, b)=0$. Taking $\zeta$ from Eq. (88) one can see that $\phi=$ $\phi_{0}\left(r^{2} Q_{s}(Y, b)\right)^{1-\gamma_{c r}}$ providing the matching of the solution 
at $\tau=r^{2} Q_{s}^{2}(Y, b)>1$ with the solution at $\tau \leq 1$ (see Sec. III B).

For large $\phi$ Eq. (91) leads to $\phi=$ Const $\exp \left(-\zeta^{2} / 8\right)$, which is the scattering amplitude of Eq. (81) for our simplified BFKL kernel. However, we find that $\Phi=1$ in this solution.

Therefore, in this simplified version of the nonlinear equation we found, that the scattering amplitude in the saturation region has geometric scaling behavior, being a function of one variable $\tau=r^{2} Q^{2}(Y, b)$ with

$$
\begin{aligned}
Q_{s}^{2}(Y, b)= & Q_{s}^{2}(Y=0) \\
& \times \exp \left(\bar{\alpha}_{S} \frac{\chi\left(\gamma_{c r}\right)}{1-\gamma_{c r}} Y-\frac{3}{4\left(1-\gamma_{c r}\right)}\left(\frac{b^{4}}{4 \alpha_{\mathrm{eff}}^{\prime 2} Y}\right)^{1 / 3}\right) .
\end{aligned}
$$

Hence we find that the $b$ dependence is concentrated in the dependence of the saturation scale, as it has been assumed in the numerous attempts to introduce $b$-dependence in the saturation models (see Refs. [50,51] and references therein).

\section{THE FROISSART DISC IN QCD}

In Secs. III C and III D we showed, that (i) the scattering amplitude shows geometric scaling behavior being a function of one variable $\zeta$ [see Eq. (80)] and (ii) $1-N(\zeta)=\Delta(\zeta) \ll 1$ for $\zeta \geq 1$. The condition $\zeta \geq 1$ means that

$$
\bar{\alpha}_{S} \frac{\chi\left(\gamma_{c r}\right)}{1-\gamma_{c r}} Y+\xi \geq \frac{3}{4\left(1-\gamma_{c r}\right)}\left(\frac{b^{4}}{4 \alpha_{\mathrm{eff}}^{\prime 2} Y}\right)^{1 / 3}
$$

Therefore, for

$$
\frac{b}{\sqrt{\alpha_{\mathrm{eff}}^{\prime}}} \leq R_{\mathrm{F}}=\left(4 Y\left(4 \bar{\alpha}_{S} \frac{\chi\left(\gamma_{c r}\right)}{3} Y+\frac{4\left(1-\gamma_{c r}\right)}{3} \xi\right)^{3}\right)^{1 / 4} .
$$

where $R_{F}$ denotes the radius of the Froissart disc.

In Fig. 3 we plot the solution to the leading twist nonlinear equation (see previous section) as function of $b$. The solution demonstrates all features of the Froissart disc with a radius, which is proportional to $Y$. The edge of the disc decreases as $\exp \left(-C_{1}\left(b-R_{F}\right)^{3}\right)$, where $C_{1}$ is a constant that can be calculated. Indeed, we can see this by plugging in the expression for the amplitude $N(r, Y ; b)=$ $N_{0}\left(r^{2} Q_{s}^{2}(Y, b)\right)^{\bar{\gamma}} \boldsymbol{b}=\boldsymbol{R}_{F}+\boldsymbol{\Delta} \boldsymbol{b}$ and expanding the amplitude with respect to $\Delta b$. One can see, that for $\Delta b \ll R_{F}$, the amplitude is equal

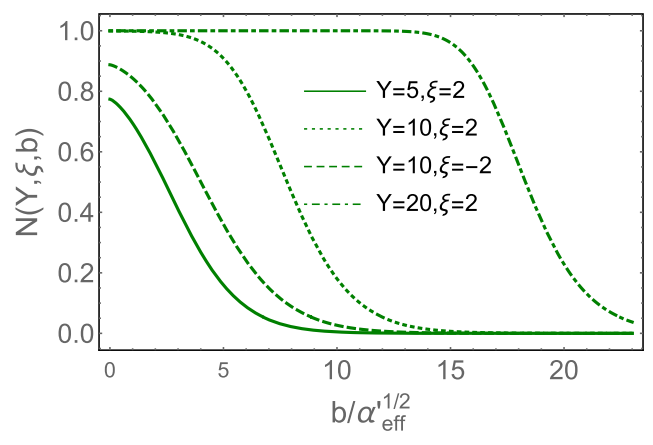

FIG. 3. The impact parameter $(b)$ dependence of the scattering amplitude. For negative $\zeta$ of Eq. (80) we use the solution of Eq. (75), while for $\zeta>0$ Eq. (91) is solved with $C(Y, b)=0$ and Const $=0 . N_{0}$ is taken to be equal to 0.055 and $\bar{\alpha}_{S}=0.25$.

$$
\begin{aligned}
N(r, Y ; b) & =N_{0} \exp \left(-\frac{3}{2}\left(\frac{2 R_{F}}{Y}\right)^{1 / 3} \frac{\Delta b}{\sqrt{\alpha_{\text {eff }}^{\prime}}}\right) \\
& \stackrel{Y \gg \xi}{\longrightarrow} N_{0} \exp \left(-6\left(\frac{\chi\left(\gamma_{c r}\right)}{3}\right)^{3 / 4} \frac{\Delta b}{\sqrt{\alpha_{\text {eff }}^{\prime}}}\right)
\end{aligned}
$$

For large $b \gg R_{F}$ the typical $b^{2} \propto \sqrt{Y}$.

The new equation leads to restoration of the Froissart theorem. Indeed, in Eq. (6) one can see, that we can use Eq. (74) and Eq. (75) to estimate the value of $b_{0}$. Indeed, this value we can find from the following equation:

$$
N\left(Y, \xi, b_{0}\right)=\underbrace{e^{\bar{\alpha}_{S} \chi\left(\gamma_{c r}\right) Y+\left(1-\gamma_{c r}\right) \xi-\frac{3}{4}\left(\frac{b_{0}^{4}}{4 \alpha^{\prime 2}{ }_{\text {eff }} Y}\right)^{1 / 3}}}_{\text {in vicinity of the saturation scale }}=f_{0} .
$$

Assuming $Y \gg \xi$ we obtain

$$
\frac{b_{0}}{\sqrt{\alpha_{\text {eff }}^{\prime}}}=\left(\frac{4 \bar{\alpha}_{S} \chi\left(\gamma_{c r}\right)}{3}\right)^{1 / 4} Y
$$

Plugging this value for $b_{0}$ into Eq. (6) we have

$$
\sigma_{\mathrm{tot}} \leq 2 \pi b_{0}^{2}=2 \pi \alpha_{\mathrm{eff}}^{\prime}\left(\frac{4 \bar{\alpha}_{S} \chi\left(\gamma_{c r}\right)}{3}\right)^{1 / 2} Y^{2}
$$

and the integral over $b>b_{0}$ gives a smaller contribution, which is of the order of $\sqrt{Y}$. Equation (98) is the Froissart theorem in the specific realization for the QCD approach, based on the new nonlinear evolution equation.

For applications one can use the simple analytical form of the solution to the nonlinear equation, given in Ref. [52]:

$N(\zeta)=a\left(1-\exp \left(-N_{0} e^{\frac{1}{2} \zeta}\right)\right)+(1-a) \frac{N_{0} e^{\frac{1}{2} \zeta}}{1+N_{0} e^{\frac{1}{2} \zeta}}$

which describes the numerical solution within the accuracy of $4 \%$ (see Fig. 4). 


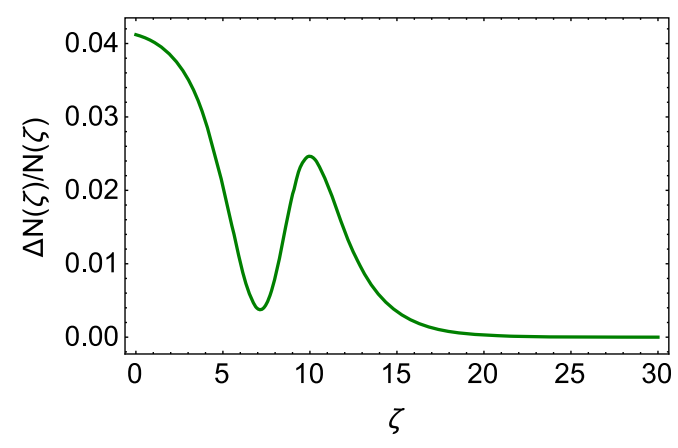

FIG. 4. The comparison of the approximate analytical formula of Eq. (99) with the numerical solution of Eq. (91). $\Delta N=$ $(N(\zeta$, Eq.(91)). $-N(\zeta$, Eq.(99))) $/ N(\zeta$, Eq.(99)). Equation (91) is solved with $C(Y, b)=0$ and Const $=0 . N_{0}$ is taken to be equal to 0.055 and $\bar{\alpha}_{S}=0.25$. In Eq. (99) $a=0.65$.

\section{CONCLUSIONS}

The main result of this paper is the new nonlinear evolution equation which includes the random walk, both in the transverse momentum and in impact parameter of the produced gluons (dipoles). We showed, that the solution to new equation results in the exponential decrease of the scattering amplitude at large impact parameter and in the restoration of the Froissart theorem. Therefore, this equation solves the long standing problem of the CGC/ saturation approach. We demonstrated, that the new equation generates the amplitude, which approaches 1 for $b \leq$ Const $Y$ and which decreases as $\exp (-\mu b)$ at $b>$ Const $Y$.

We found the solution to the equation in the kinematic region, where $\mathcal{Z} \ll 4 \bar{\gamma} Y$. Hence, a problem to tackle in the future, is to develop a more general solution, as well as to describe the available experimental data within an approach, based on the new equation.

\section{ACKNOWLEDGMENTS}

We thank our colleagues at Tel Aviv University and UTFSM for encouraging discussions. This research was supported by CONICYT PIA/BASAL FB0821(Chile) and Fondecyt (Chile) Grant No. 1180118.
[1] Y. V. Kovchegov and E. Levin, Quantum Choromodynamics at High Energies, Cambridge Monographs on Particle Physics, Nuclear Physics and Cosmology (Cambridge University Press, Cambridge, England, 2012) and references therein.

[2] V. S. Fadin, E. A. Kuraev, and L. N. Lipatov, On the pomeranchuk singularity in asymptotically free theories, Phys. Lett. 60B, 50 (1975); E. A. Kuraev, L. N. Lipatov, and V.S. Fadin, The Pomeranchuk singularity in nonabelian gauge theories, Zh. Eksp. Teor. Fiz. 72, 377 (1977) [Sov. Phys. JETP 45, 199 (1977)]; I. I. Balitsky and L. N. Lipatov, The Pomeranchuk singularity in quantum chromodynamics, Yad. Fiz. 28, 1597 (1978) [Sov. J. Nucl. Phys. 28, 822 (1978)].

[3] L. N. Lipatov, The bare Pomeron in quantum chromodynamics, Zh. Eksp. Teor. Fiz. 90, 1536 (1986) [Sov. Phys. JETP 63, 904 (1986)].

[4] M. Froissart, Asymptotic behavior and subtractions in the Mandelstam representation, Phys. Rev. 123, 1053 (1961); A. Martin, Scattering Theory: Unitarity, Analitysity and Crossing, Lecture Notes in Physics (Springer-Verlag, Berlin, Heidelberg, New-York, 1969).

[5] E. M. Levin and M. G. Ryskin, High-energy hadron collisions in QCD, Phys. Rep. 189, 268 (1990).

[6] E. M. Levin and M. G. Ryskin, The shrinkage of the diffraction peak of the vare Pomeron in QCD, Sov. J. Nucl. Phys. 50, 881 (1989) [Yad. Fiz. 50, 1417 (1989)] [Z. Phys. C 48, 231 (1990)].

[7] E. Levin and C. I. Tan, Heterotic pomeron: A unified treatment of high-energy hadronic collisions in QCD, in Santiago de Compostela, Proceedings, Multiparticle dynamics (1992), pp. 568-575; Fermilab Batavia, FERMILAB Report
No. FERMILAB-Conf-92-391, 1993. p. 9; Brown University Providence Report No. BROWN-HET-889, 1993.

[8] D. Y. Ivanov, R. Kirschner, E. M. Levin, L. N. Lipatov, L. Szymanowski, and M. Wusthoff, The BFKL pomeron in (2+ 1)-dimensional QCD, Phys. Rev. D 58, 074010 (1998).

[9] D. Kharzeev and E. Levin, Scale anomaly and 'soft' pomeron in QCD, Nucl. Phys. B578, 351 (2000).

[10] D. E. Kharzeev, Y. V. Kovchegov, and E. Levin, QCD instantons and the soft pomeron, Nucl. Phys. A690, 621 (2001).

[11] A. Kovner and U. A. Wiedemann, Nonlinear QCD evolution: Saturation without unitarization, Phys. Rev. D 66, 051502 (2002); Perturbative saturation and the soft pomeron, Phys. Rev. D 66, 034031 (2002); No Froissart bound from gluon saturation, Phys. Lett. B 551, 311 (2003).

[12] E. Ferreiro, E. Iancu, K. Itakura, and L. McLerran, Froissart bound from gluon saturation, Nucl. Phys. A710, 373 (2002).

[13] K. J. Golec-Biernat and A. M. Stasto, On solutions of the Balitsky-Kovchegov equation with impact parameter, Nucl. Phys. B668, 345 (2003).

[14] S. Bondarenko, E. Levin, and C. I. Tan, High energy amplitude as an admixture of 'soft' and 'hard' pomerons, Nucl. Phys. A732, 73 (2004).

[15] E. Gotsman, M. Kozlov, E. Levin, U. Maor, and E. Naftali, Towards a new global QCD analysis: Solution to the nonlinear equation at arbitrary impact parameter, Nucl. Phys. A742, 55 (2004).

[16] Y. Hatta and A. H. Mueller, Correlation of small-x gluons in impact parameter space, Nucl. Phys. A789, 285 (2007).

[17] A.H. Mueller and S. Munier, Correlations in impactparameter space in a hierarchical saturation model for QCD at high energy, Phys. Rev. D 81, 105014 (2010). 
[18] J. Berger and A. M. Stasto, Small x nonlinear evolution with impact parameter and the structure function data, Phys. Rev. D 84, 094022 (2011).

[19] J. Berger and A. Stasto, Numerical solution of the nonlinear evolution equation at small $\mathrm{x}$ with impact parameter and beyond the LL approximation, Phys. Rev. D 83, 034015 (2011).

[20] A. Kormilitzin and E. Levin, Non-linear equation: Energy conservation and impact parameter dependence, Nucl. Phys. A849, 98 (2011).

[21] E. Levin and S. Tapia, BFKL Pomeron: Modeling confinement, J. High Energy Phys. 07 (2013) 183.

[22] E. Levin, L. Lipatov, and M. Siddikov, BFKL Pomeron with massive gluons, Phys. Rev. D 89, 074002 (2014).

[23] E. Levin, Large $\boldsymbol{b}$ behaviour in the CGC/saturation approach: BFKL equation with pion loops, Phys. Rev. D 91, 054007 (2015).

[24] O. V. Kancheli, On the parton picture of Froissart asymptotic behavior, arXiv:1609.07657.

[25] J. Cepila, J. G. Contreras, and M. Matas, Collinearly improved kernel suppresses Coulomb tails in the impactparameter dependent Balitsky-Kovchegov evolution, Phys. Rev. D 99, 051502 (2019).

[26] D. Bendova, J. Cepila, J. G. Contreras, and M. Matas, Solution to the Balitsky-Kovchegov equation with the collinearly improved kernel including impact-parameter dependence, Phys. Rev. D 100, 054015 (2019).

[27] C. Contreras, E. Levin, and R. Meneses, BFKL equation in the next-to-leading order: Solution at large impact parameters, Eur. Phys. J. C 79, 842 (2019).

[28] E. Gotsman and E. Levin, Large impact parameter behaviour in the $\mathrm{CGC} /$ saturation approach: A new non-linear equation, arXiv:1910.11662.

[29] P. D. B. Collins, An Introduction to Regge Theory and HighEnergy Physics, Cambridge Monographs on Mathematical Physics (Cambridge University Press, Cambridge, England, 2009), p. 460.

[30] Regge Theory of Low pT Hadronic Interaction, edited by L. Caneschi (North-Holland, Amsterdam, 1989).

[31] V. N. Gribov, Strong Interactions of Hadrons at High Energies (Cambridge University Press, Cambridge, England, 2008); The Theory of Complex Angular Momenta: Gribov Lectures on Theoretical Physics, Cambridge Monographs on Mathematical Physics (Cambridge University Press, Cambridge, England, 2003), p. 312; Space-time description of hadron interactions at high-energies, arXiv: hep-ph/0006158; Inelastic processes at super high-energies and the problem of nuclear cross-sections, Yad. Fiz. 9, 640 (1969) [Sov. J. Nucl. Phys. 9, 369 (1969)].

[32] E. Levin, Everything about Reggeons. Part 1: Reggeons in 'soft' interaction, arXiv:hep-ph/9710546.

[33] R. P. Feynman, Very High-Energy Collisions of Hadrons, Phys. Rev. Lett. 23, 1415 (1969); Photon-Hadron Interactions (CRC Press, Boca Raton, 1972).

[34] F. E. Low, A model of the bare Pomeron, Phys. Rev. D 12, 163 (1975); S. Nussinov, Colored Quark Version of Some Hadronic Puzzles, Phys. Rev. Lett. 34, 1286 (1975).

[35] A. H. Mueller, $\mathrm{O}(2,1)$ analyses of single particle spectra at high energy, Phys. Rev. D 2, 2963 (1970).
[36] A. H. Mueller, Soft gluons in the infinite momentum wave function and the BFKL Pomeron, Nucl. Phys. B415, 373 (1994); Unitarity and the BFKL pomeron, Nucl. Phys. B437, 107 (1995).

[37] L. V. Gribov, E. M. Levin, and M. G. Ryskin, Semihard processes in QCD, Phys. Rep. 100, 1 (1983).

[38] A. H. Mueller and J. Qiu, Gluon recombination and shadowing at small values of $x$, Nucl. Phys. B268, 427 (1986).

[39] L. McLerran and R. Venugopalan, Computing quark and gluon distribution functions for very large nuclei, Phys. Rev. D 49, 2233 (1994); Gluon distribution functions for very large nuclei at small transverse momentum, Phys. Rev. D 49, 3352 (1994); Green's function in the color field of a large nucleus, Phys. Rev. D 50, 2225 (1994); Fock space distributions, structure functions, higher twists, and small $x$, Phys. Rev. D 59, 094002 (1999).

[40] I. Balitsky, Operator expansion for high-energy scattering, Nucl. Phys. B463, 99 (1996); Factorization and high-energy effective action, Phys. Rev. D 60, 014020 (1999); Y. V. Kovchegov, Small-x $F_{2}$ structure function of a nucleus including multiple Pomeron exchanges, Phys. Rev. D 60, 034008 (1999).

[41] A. D. Polyanin, Handbook of Linear Partial Differential Equations for Engineers and Scientists (Chapman \& Hall/ CRC, Boca Raton, London, New York, Washington, D.C., 2001).

[42] A. A. Anselm and V. N. Gribov, Zero pion mass limit in interactions at very high-energies, Phys. Lett. B 40, 487 (1972).

[43] I. Gradstein and I. Ryzhik, Table of Integrals, Series, and Products (Academic Press, London, 1994), 5th ed.

[44] A. H. Mueller and D. N. Triantafyllopoulos, The energy dependence of the saturation momentum, Nucl. Phys. B640, 331 (2002).

[45] J. Bartels and E. Levin, Solutions to the Gribov-LevinRyskin equation in the nonperturbative region, Nucl. Phys. B387, 617 (1992).

[46] E. Levin and K. Tuchin, Solution to the evolution equation for high parton density QCD, Nucl. Phys. B573, 833 (2000).

[47] A. M. Stasto, K. J. Golec-Biernat, and J. Kwiecinski, Geometric Scaling for the Total Gamma* p Cross-Section in the Low x Region, Phys. Rev. Lett. 86, 596 (2001).

[48] E. Iancu, K. Itakura, and L. McLerran, Geometric scaling above the saturation scale, Nucl. Phys. A708, 327 (2002).

[49] V. N. Gribov and L. N. Lipatov, Deep inelastic e p scattering in perturbation theory, Yad. Fiz. 15, 781 (1972) [Sov. J. Nucl. Phys. 15, 438 (1972)]; G. Altarelli and G. Parisi, Asymptotic freedom in parton language, Nucl. Phys. B126, 298 (1977); Y. L. Dokshitzer, Calculation of the structure functions for deep inelastic scattering and e+e- annihilation by perturbation theory in quantum chromodynamics, Zh. Eksp. Teor. Fiz. 73, 1216 (1977) [Sov. Phys. JETP 46, 641 (1977)].

[50] A. H. Rezaeian and I. Schmidt, Impact-parameter dependent color glass condensate dipole model and new combined HERA data, Phys. Rev. D 88, 074016 (2013).

[51] C. Contreras, E. Levin, and I. Potashnikova, CGC/saturation approach: A new impact-parameter dependent model, Nucl. Phys. A948, 1 (2016).

[52] E. Levin, Dipole-dipole scattering in CGC/saturation approach at high energy: Summing Pomeron loops, J. High Energy Phys. 11 (2013) 039. 